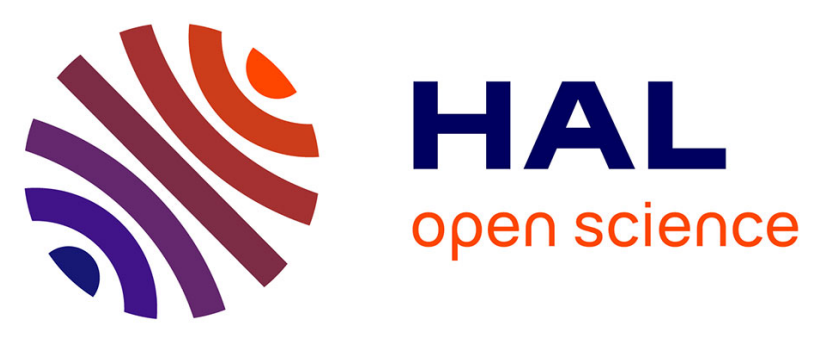

\title{
Revisiting the human uncinate fasciculus, its subcomponents and asymmetries with stem-based tractography and microdissection validation
}

Janice Hau, Silvio Sarubbo, Jean Christophe Houde, Francesco Corsini, Gabriel Girard, Charles Deledalle, Fabrice Crivello, Laure Zago, Emmanuel Mellet, Gaël Jobard, et al.

\section{To cite this version:}

Janice Hau, Silvio Sarubbo, Jean Christophe Houde, Francesco Corsini, Gabriel Girard, et al.. Revisiting the human uncinate fasciculus, its subcomponents and asymmetries with stem-based tractography and microdissection validation. Brain Structure and Function, 2017, 222, pp.1645-1662. 10.1007/s00429-016-1298-6 . hal-01382592

\section{HAL Id: hal-01382592 https://hal.science/hal-01382592}

Submitted on 5 Feb 2021

HAL is a multi-disciplinary open access archive for the deposit and dissemination of scientific research documents, whether they are published or not. The documents may come from teaching and research institutions in France or abroad, or from public or private research centers.
L'archive ouverte pluridisciplinaire HAL, est destinée au dépôt et à la diffusion de documents scientifiques de niveau recherche, publiés ou non, émanant des établissements d'enseignement et de recherche français ou étrangers, des laboratoires publics ou privés. 


\title{
Revisiting the human uncinate fasciculus, its subcomponents and asymmetries with stem-based tractography and microdissection validation
}

\author{
Janice HAU ${ }^{1}$, Silvio SARUBBO ${ }^{2,3}$, Jean Christophe HOUDE ${ }^{4}$, Francesco CORSIN| ${ }^{2,3}$, \\ Gabriel GIRARD ${ }^{4}$, Charles DELEDALLE ${ }^{5}$, Fabrice CRIVELLO ${ }^{1}$, Laure ZAGO¹, \\ Emmanuel MELLET ${ }^{1}$, Gaël JOBARD ${ }^{1}$, Marc JOLIOT ${ }^{1}$, Bernard MAZOYER ${ }^{1}$, \\ Nathalie TZOURIO-MAZOYER ${ }^{1}$, Maxime DESCOTEAUX ${ }^{4}$, Laurent PETIT ${ }^{1}$
}

${ }^{1}$ Groupe d'Imagerie Neurofonctionnelle, Institut des Maladies Neurodégénératives - UMR 5293, CNRS, CEA University of Bordeaux, Bordeaux, France

2 Division of Neurosurgery, Department of Neurosciences, "S. Chiara” Hospital, Trento APSS, Italy

${ }^{3}$ Structural and Functional Connectivity Lab, Division of Neurosurgery, "S. Chiara" Hospital, Trento APSS, Italy

${ }^{4}$ Sherbrooke Connectivity Imaging Lab, University of Sherbrooke, Canada

${ }^{5}$ Institut de Mathématiques de Bordeaux - UMR 5251, CNRS, Talence, France

Corresponding author:

Laurent PETIT, Groupe d'Imagerie Neurofonctionnelle (GIN) - IMN - UMR5293

Université de Bordeaux, PAC Carreire

146 rue Léo Saignat - CS 61292- Case 28

33076 Bordeaux cedex

Phone: +33 547304398

Fax: +33547304394

Email: laurent.petit@u-bordeaux.fr 


\section{Abstract}

Despite its significant functional and clinical interest, the anatomy of the uncinate fasciculus (UF) has received little attention. It is known as a 'hook-shaped' fascicle connecting the frontal and anterior temporal lobes and is believed to consist of multiple subcomponents. But knowledge of its precise connectional anatomy in humans is lacking and its subcomponent divisions are unclear. In the present study we evaluate the anatomy of the UF and provide its detailed normative description in 30 healthy subjects with advanced particle-filtering tractography with anatomical priors and robustness to crossing fibers with constrained spherical deconvolution. We extracted the UF by defining its stem encompassing all streamlines that converge into a compact bundle, which consisted not only of the classic hook-shaped fibers but also straight horizontally oriented. We applied an automatic clustering method to subdivide the UF bundle and revealed five subcomponents in each hemisphere with distinct connectivity profiles, including different asymmetries. A layer-by-layer microdissection of the ventral part of the external and extreme capsules using Klingler's preparation also demonstrated five types of uncinate fibers that, according to their pattern, depth, and cortical terminations, were consistent with the diffusion-based UF subcomponents. The present results shed new light on the UF cortical terminations and its multicomponent internal organization with extended cortical connections within the frontal and temporal cortices. The different lateralization patterns we report within the UF subcomponents reconcile the conflicting asymmetry findings of the literature. Such results clarifying the UF structural anatomy lay the groundwork for more targeted investigations of its functional role, especially in semantic language processing. 


\section{Keywords}

White matter anatomy

Uncinate fasciculus

Healthy human

Brain asymmetry

Diffusion imaging tractography

Klingler's dissection

\section{Acknowledgments}

This study belongs to the project TRAIL\&TRACKS supported by a public grant from the French Agence Nationale de la Recherche within the context of the Investments for the Future Program, referenced ANR-10-LABX-57 and named TRAIL.

$\mathrm{JH}$ was also partly supported by the ANR (Agence Nationale de la Recherche) funded project BIOMIST (no. ANR-13-CORD-0007). 


\section{Introduction}

Recent literature has revealed that the definitions of many of the fiber pathways based on 19th century dissection studies are steeped in controversy and confusion (Forkel et al., 2014; Yeatman et al., 2014; Meola et al., 2015). Some of these debates are resurfacing about the area of white matter passage known as the external and extreme capsules, which may have important implications for understanding language function (Axer et al., 2013; Bajada et al., 2015; Mars et al., 2015). In the present study, we re-evaluate the descriptive anatomy of the uncinate fasciculus (UF), one of the association pathways that passes through these capsules, that has been of significant interest in clinical disorders such as psychopathy, Alzheimer's disease, schizophrenia and major depression (Price et al., 2008; Yasmin et al., 2008; Craig et al., 2009; Phan et al., 2009; Steffens et al., 2011; Hernando et al., 2015; Wong et al., 2015) and various brain functions/states including language processing, empathy and associative learning (Parker et al., 2005; Duffau et al., 2009; Papagno et al., 2011; Von Der Heide et al., 2013; Oishi et al., 2015; Thomas et al., 2015).

The UF was first described by Johann Christian Reil who named it ('Hakenbündel' in the original text), as a pathway that "encircles the gap by which the frontal lobe is separated from the temporal lobe, and is constituted by the fan-like expansions of the central fibers of the gyri of the frontal and temporal lobes that aggregate from both sides in one stem "(p. 200, Reil, 1809, translation from (Schmahmann and Pandya, 2006)). Karl Burdach expanded on Reil's description, observing fibers that curve laterally from the external capsule to the frontal lobe "into its lateral areas as well as into the lateral portions of the basal areas" (p.152, Burdach, 1822, translation from (Schmahmann and Pandya, 2006)), and gave it its current Latin name. Over the next two centuries most studies confirmed their classical hook-shaped descriptions (Ebeling and von Cramon, 1992; Ture et al., 2000; Kier et al., 2004; Travers, 2008; Martino et al., 2011). However, there have been some early descriptions that have contrasted with the UF's classic hook-like shape. Dejerine, who referenced Burdach's work 80 years later, described the UF as having not only the characteristic hook-shaped fibers but 
fibers that become straight and may even curve in the inverse direction (Dejerine and Dejerine-Klumpke, 1895). In the same vein, early studies described that some UF fibers present an horizontal course linking the orbital part of the frontal cortex to the middle to posterior part of the temporal lobe (Gordinier, 1899; Testut, 1900). Both Gordinier and Testut proposed specific subcomponents of the UF whereas Dejerine did not make the differentiation. In their study, which remains the reference today to the defintion of the human UF, Ebeling and von Cramon (1992) described it as a curved dumb-bell with a thin fan-like arrangement of fibers between the orbital gyri of the frontal cortex and the tip of the temporal lobe.

Diffusion imaging tractography has been applied to the anatomical study of white matter and has been able to resolve some of the debates surrounding fiber tract anatomy, such as the existence or inexistence of tracts and their termination territories (Catani et al., 2002; Forkel et al., 2014; Yeatman et al., 2014; Meola et al., 2015; Hau et al., 2016). By applying an original anatomical stem-based method to virtually dissect the UF, we have thereby recently revealed a map of the cortical UF termination that consistently shown terminations in the temporal cortex, beyond the temporal pole (Hau et al., 2016). But a more detailed anatomical description, such as putative UF subcomponents, was limited by the use of diffusion tensor model for whole-brain tractography.

In the present study we aim to address the above questions by re-evaluating the anatomy of the UF in 30 subjects using diffusion imaging tractography with constrained spherical deconvolution (CSD) particle-filter tracking (PFT) and anatomical priors. CSD-PFT has shown to be able to address many of the current tractography biases of length/size/curvature by including knowledge of the anatomy from the T1-weigthed images and tracking seeds placed at the WM/GM interface (Girard et al., 2014). Hence, streamlines produced by CST-PFT tracking must end in the GM, cannot stop in WM or CSF, thus overcoming many limitations of current tractography algorithms.

The whole UF was therefore extracted by applying our approach based on the delineation of the stem of the bundle from the whole-brain tractogram (Hau et al., 2016). This approach is 
aimed at reproducing the definition of the fascicle introduced by the early dissectionists, who consequently delineated fiber tracts by identifying them at their stem before following the fibers to see where they terminate. We also for the first time applied a clustering approach to divide the UF bundle into subcomponents without any a priori on the number of subcomponents. In order to validate the anatomical findings observed from tractography, we additionally performed post-mortem dissection of the UF in 4 specimens. A lateral to medial layer-by-layer approach was taken to reveal the specific connections in relation to their topographical organization (i.e., we present the connections first observed in the most superficial layer progressing to the deepest layer).

\section{Methods}

\section{Image acquisition and tractography}

The present study uses diffusion-weighted and T1-weighted images of 30 healthy right-handed subjects from the BIL\&GIN database (Mazoyer et al., 2016) and selected as typical (left-lateralized) subjects from fMRI of language sentence production (15 female, mean age $=26.3$ years, age range $=22-34$ years) (details in (Mazoyer et al., 2016)). All subjects gave written consent to participate in the study, which was approved by the local ethics committee (CCPRB Basse-Normandie).

The diffusion-weighted images were processed using the MRtrix software package (http://www.brain.org.au/software/). First the diffusion tensor and fractional anisotropy (FA) maps were created. A single fiber response function was estimated from FA values above 0.7 and used as input for constrained spherical deconvolution (CSD) (Tournier et al., 2007; Descoteaux et al., 2009) for computing the fiber orientation distribution functions (fODF), with a spherical harmonic order of 6 . The processed datasets (fODF, FA, RGB maps) were upsampled to $1 \mathrm{~mm}^{3}$ spatial resolution using trilinear interpolation (Tournier et al., 2012; Girard et al., 2014). Fiber tracking was performed on the upsampled fODF maps using 
particle-filter tractography with anatomical priors (Girard et al., 2014), creating a whole-brain tractogram (three-dimensional reconstruction of the white matter structure) for each subject. Girard et al.'s tracking algorithm is based on a continuous map criterion (CMC) to keep only the streamlines that are seeded in the white/grey-matter interface and terminate in the grey matter, based on the tissue partial volume estimation maps processed from the subject's T1 image. To create the tissue partial volume estimation maps, the T1 was first warped to the diffusion space using ANTS linear and non-linear registration (http://stnava.github.io/ANTs/). The T1 was segmented into regions of grey matter, white matter and cerebrospinal fluid using FSL's (http://www.fmrib.ox.ac.uk/fsl/index.html) fast tool and the partial volume (include and exclude, see Girard et al., 2014) maps were created based on these segmentations. In addition, to optimize the stopping criteria, this method uses particle filtering, which applies a backtracking step to find alternative valid pathways to continue the tracking. It allowed reducing premature stopping in areas of low anisotropy. Seeding was initiated with 10 seeds per voxel in the white/grey-matter interface generating a whole brain tractogram of approximately 1.5 million of streamlines. A length threshold was set at a minimum of 10 and maximum of $250 \mathrm{~mm}$. If not otherwise specified the default parameters were used.

The Johns Hopkins University (JHU) template (Oishi et al., 2009) was modified to individualize the temporal pole within the temporal lobe regions, and was used to automatically parcellate the brain into cortical regions (Hau et al., 2016). The template was first warped from the standard space to the native diffusion space of each subject through linear and non-linear registration with ANTS. A subset of the template was created that included all of the grey matter regions (i.e., cortical and subcortical, including JHU template superficial white matter regions which were merged with their corresponding gyral region). These parcellations were used as regions of interest (ROIs) to filter the streamlines and determine their termination regions for the connectivity analysis. 


\section{Stem-based anatomical virtual dissection of UF}

The UF was extracted by performing a stem-based anatomical virtual dissection (Hau et al., 2016) in each hemisphere of the 30 whole-brain tractograms in the subject's diffusion space (Fig. 1A). We first took into account only streamlines with one cortical termination in the frontal lobe and the other in the temporal, parietal, and occipital lobes, all lobes constituted with the modified JHU template (Fig. 1B). The UF was easily identifiable as the compact bundle of streamlines with a characteristic hook-shaped trajectory connecting the frontal and temporal lobes (Fig 1C). A rectangular ROI was drawn on a single coronal section covering the entire stem with a margin of a few voxels and used to extract a set of candidate UF streamlines (Fig. 1C, zoom). Note that only a small percentage of the streamlines were first visualized $(10 \%)$ in order to reduce computational demands given the large size of the whole-brain tractograms. Once the ROI filter was applied all the streamlines were visualized to check that the UF stem included the entire bundle, or otherwise it was modified interactively (Fig 1D). We removed adjacent IFOF streamlines still passing through the UF stem by placing a coronal slice filter where there was a break separating UF from IFOF posterior temporal courses (Fig. 1E). All remaining streamlines passing through the UF stem and connecting the frontal lobe with the temporal (or limbic) cortical areas were therefore considered as UF streamlines (Fig. 1F). We finally applied an outlier removal algorithm (Côté et al., 2015) that used hierarchical QuickBundles clustering (Garyfallidis et al., 2012) to prune anatomical outlier streamlines (cut-off at 20\%), namely streamlines 'flitting around' a direct UF projection with multiple curves and loops between their frontal and temporal terminations (Fig. 1G). Visualization, streamline filtering and the manual delineation of the UF stem were performed with the software TrackVis (http://www.trackvis.org/).

Each UF stem was registered to the MNI space using the inverse warp and affine matrices that were used to register the anatomical template to the diffusion space in order to report their mean stem volume as the total amount of $1 \mathrm{~mm}^{3}$-voxels and center of mass coordinates in each hemisphere. 


\section{Automatic segmentation of UF subcomponents}

We used a clustering method to automatically classify the UF bundles into subcomponents. A twelve-dimensional Gaussian mixture model was trained to encode the joint distribution of the locations and orientations of the pairs of streamline endpoints (two locations + two directions in three-dimensional space) with the expectation maximization algorithm (Dempster et al., 1976). Each ending orientation was measured between the endpoint and the point located at $10 \%$ from it with respect to the total length. Each Gaussian of the model represents the distribution of endpoints in one cluster that can be visualized with a pair of ellipses (Fig. 2). The number of clusters was automatically selected based on the Bayesian Information Criterion (Schwartz, 1978). Clusters with less than 25 streamlines were discarded and the streamlines in these groups were reassigned to the remaining clusters. A streamline could therefore only be assigned to one cluster.

Clustering was performed on the entire set of UF streamlines across all subjects and hemispheres. The UFs were transformed to MNI space using the same inverse warp and affine matrices that for the UF stem with tract registration tools in Tract Querier (Wassermann et al., 2013) and merged in their respective hemispheres. The right UF dataset was flipped on the $\mathrm{x}$-axis and combined with the left UF dataset to create a full UF dataset. Once the clusters were finalized on the full dataset, they were applied to the merged datasets of each hemisphere, re-flipping on the x-axis for the right hemisphere. The subcomponents were attributed to each subject in their native diffusion space.

As the output of such clustering depends on the choice of an initialization, we ran the clustering three additional times with random initialization. To assess the reliability of the clusters between runs, we calculated Jaccard indexes to determine the degree of overlap between the subcomponents obtained in the first run and those in the additional runs. The Jaccard index measures similarity between finite sample sets, and is defined as the size of the intersection divided by the size of the union of the sample sets. 
In order to get a better interpretation of all such clusters in terms of trajectory we computed the average and covariance of streamlines in each cluster and represented both statistics as a three-dimensional wireframe (Figure 2).

\section{Analysis of UF subcomponents}

We first assessed the frequency of presence of each subcomponent across the group. A subcomponent was considered to be present in a subject's hemisphere if it passed a threshold of $2 \%$ of the total number of UF streamlines extracted in this hemisphere. We therefore computed the number of streamlines, voxel-based volume and mean streamlines length to assess every subcomponent properties (Table 2).

The number of streamlines connecting a pair of regions in a UF subcomponent can be tallied to provide a measure of tract density. It was estimated for a subcomponent by calculating a streamline score as the number of streamlines normalized by the total number of left and right streamlines and weighted by the mean streamlines length to account for biases in the tracking distance. The tallies were obtained using the UCLA Multimodal Connectivity Software Package (http://ccn.ucla.edu/wiki/index.php) with the ROls of the modified JHU template. The distribution of paired connections in each subcomponent was investigated by calculating a mean streamline score only on the subjects in whom the subcomponent was present.

To assess laterality effects for the number of streamlines and volume, asymmetry indexes were calculated on each subcomponent using the following formula: $\frac{\text { Right-Left }}{\text { Right+Left }}$. Twotailed t-tests using the Bonferroni correction $(p<0.05)$ were used to test for asymmetry with a null hypothesis of 0 . Linear least squares effects of age, gender and brain volume were also tested.

To understand the relative organization of the subcomponents, we obtained the mean and individual center of mass coordinates of each subcomponent at the stem. We binarized the subcomponents to create a volume and then extracted the intersection between the stem and the subcomponent in the subject's diffusion space. The intersections were registered to 
$\mathrm{MNI}$ space using the same registration process was done for the stems and their centers of mass were calculated. Repeated measures ANOVA tests were used to assess global differences in the center of mass locations across subcomponents. If significant, these were followed by two-tailed t-tests to assess differences in the $x, y$ and $z$ coordinates of each subcomponent with respect to their closest subcomponent.

\section{Post-mortem dissection of UF}

Four left hemispheres were treated with $10 \%$ formalin, according to a modified preparation of Klingler's technique, as previously described (Sarubbo et al., 2015). Blunt microdissection (x 5) was performed with wooden spatulas, according with Klingler's cortex sparing techniques, as previously described (Sarubbo et al., 2013; Sarubbo et al., 2015). In the first step we removed the cortices of the frontal lobe and of the anterior two-thirds of the temporal lobe. In the second step the short U-fibers of the frontal and temporal regions were removed. Then we proceeded to remove the gray matter of the long and short gyri of the insula. Finally, after localization of the frontal and temporal opercula we removed the WM of the extreme capsule and claustrum, demonstrating the stem of UF within the most ventral third of the external capsule (EC), at the level of limen insulae.

In all the specimens the dissection and data collection started at the limen insulae where we followed the arching fibers of UF (layer by layer) up to the terminal sites, collecting the reciprocal connectivity between frontal and temporal cortices. Distinction between UF and IFOF stems were performed considering the posterior course of the fibers composing these.

\section{Results}

\section{The stem-based uncinate fasciculus (UF)}

The UF streamlines were observed in all 30 subjects bilaterally, represented as a dense, compact and coherent bundle connecting the frontal and temporal lobes. The anatomical break between the UF and IFOF posterior courses in the temporal lobe was 
clearly observed in 57 out of 60 hemispheres. In the 3 remaining cases, the posterior terminations of the two tracts appeared to be continuous within the temporal lobe and the coronal filter was placed based on a best guess. In all cases $(\mathrm{N}=60)$, the coronal slice filter was effective in isolating the UF streamlines from the adjacent IFOF streamlines which were present at the supero-medial edge of the UF stem (Figure 1E). The mean \pm standard deviation coronal filter in $\mathrm{MNI}$ space was located at $-43 \pm 12 \mathrm{~mm}$ and $-40 \pm 13 \mathrm{~mm}$ from the vertical plane passing through the anterior commissure in the left and right hemispheres, respectively (Table 1$)$.

The mean UF stem volumes (in $\mathrm{mm}^{3}$ ) in the left and right hemispheres were $57 \pm 14$ and 63 \pm 26 , respectively (Table 1 ). The mean center of mass coordinates of the left and right UF stems were symmetrically situated between the posterior part of the putamen and the claustrum (Table 1)

\section{Subcomponents of the UF}

Five subcomponents were consistently identified across four runs of the automatic clustering method. The classified streamlines of the group data (both left and right hemispheres combined) subsampled at $5 \%$ are shown in Figure 2. The relative difference in the Gaussian mixture model parameters from run to run did not impact the clustering results. Indeed, the mean Jaccard index for the initial run with respect to the three additional runs was 1 for all subcomponents indicating that the clustering produced identical segmentation results across the four runs.

Each of the five UF subcomponents had a distinct structural pattern of trajectory and connections (Figure 3 and 4). The frequency of presence across the group for each subcomponent was superior to $80 \%$ bilaterally except for the right $\mathrm{C} 1$ subcomponent $(63 \%)$. These frequencies as well as mean number of streamlines, volume and mean length of each subcomponent are summarized in Table 2. Significant different asymmetries were observed depending on the subcomponent (Table 3). Below, we describe the region-to-region 
connections, frequencies and asymmetries of each subcomponent. They have been named of the basis of their anatomical definition and location within the UF stem.

Class 1 grouped large fan-shaped streamlines in a dorso-lateral UF subcomponent connecting the superior (SFG), middle (MFG) and inferior (IFG) frontal areas with the superior (STG), middle (MTG) and inferior (ITG) temporal gyri and in a less extent the temporal pole (Figure 3-C1 and 4-C1). Both SFG and IFG to MTG connections appear to be the most predominant. It was more frequently present in the left (27 subjects) than right hemisphere (19 subjects). Its volume was left lateralized $(p<0.01)$, due to more frequent streamlines terminating in the dorsal part of the SFG (Figure 3-C1) and, although there was a left lateralized trend for the number of streamlines $(\mathrm{Al}=-0.24)$, this did not reach the Bonferroni-corrected level of significance, controlling for age, gender and brain volume.

Class 2 grouped horizontal fan-shaped streamlines in a ventro-lateral UF subcomponent mainly connecting the orbito-frontal regions with the MTG and ITG and temporal pole (Figure 3-C2 and 4-C2). It was present in all subjects bilaterally, with no significant asymmetry.

Class 3 grouped hook-shaped streamlines in a ventro-medial UF subcomponent connecting the orbito-frontal regions with the temporal pole (Figure 3-C3 and 4-C3). The medial orbito-frontal to temporal pole connections are the most predominant. It was present in 26 subjects in the left hemisphere and in all subjects in the right hemisphere, and a right lateralized asymmetry for the number of streamlines $(p<0.001)$ and volume $(p<0.001)$, controlling for age, gender and brain volume.

Class 4 grouped short hook-shaped streamlines in a short postero-medial UF subcomponent that connects the posterior part of the orbito-frontal and rectus gyri with the temporal pole and fusiform gyrus (Figure $3-\mathrm{C} 4$ and 4-C4). The medial fronto-orbital to temporal pole connections are the most predominant. It was present in almost all subjects bilaterally (29 and 28 in the left and right hemispheres, respectively) with no significant asymmetry. 
Class 5 grouped short straight streamlines in a short antero-medial UF subcomponent that connects the orbito-frontal and rectus gyri with the most antero-medial part of the STG and ITG temporal gyri (Figure 3-C5 and 4-C5). The most predominant connections are between the medial orbito-frontal and inferior temporal gyri. It was present in all subjects in the left hemisphere and in 24 subjects in the right hemisphere, and showed a right lateralized asymmetry for volume $(p=0.004)$ only, controlling for age, gender and brain volume.

\section{Cortex-sparing UF fiber dissection}

Note first that all the sites of UF terminations reported were systematically observed in the 4 specimens dissected. Each UF dissection started from its classical anatomical definition by exposing fibers arching around the anterior and ventral third of the external and extreme capsules and connecting the temporal and frontal lobe. After the removal of the insula and claustro-opercular fibers, we proceeded from the most superficial to the deepest layers of fibers passing through the UF stem (Figure 5).

We first exposed the most superficial and lateral layer of UF fibers that closely arch around the frontal and temporal opercula. They connect the pars opercularis and triangularis of IFG with the middle third of STG and the anterior third of MTG, respectively, and the pars orbitalis of IFG and the basal fronto-orbital cortex with the antero-lateral portion of the temporal pole (Figure 5A).

A second deeper layer of fibers with a larger fan-shaped course was demonstrated to connect a large portion of the fronto-lateral cortices with the lateral convexity of the temporal cortex and the temporal pole (Figure 5B). We found bi-directional connectivity between: the middle third of STG and middle/anterior third of MTG with, respectively, the posterior third of SFG and MFG and the anterior third of the MFG; the antero-lateral portion of the temporal pole with the dorsal portion of the anterior third of IFG. Fibers of this subcomponent cover the most superficial portion of the IFOF stem (Figure 5B).

At this level, the orientation and the location of the fibers within the UF stem changed. The fibers of a third layer of the UF appeared all positioned antero-medially in respect to the 
IFOF stem (Figure 5E). These fibers have a more anterior, shorter and arched course. We found a common pattern of connectivity between the lateral temporal pole and the lateral fronto-orbital cortices and between the anterior portion of the temporal pole and the frontal pole (Figure 5C-5D).

The deepest UF fibers have the shortest and the most medial direction. Considering their different course, orientation and terminations they can be divided in a fourth and fifth layer connecting respectively (Figure 5D), the antero-medial cortices of the temporal pole with the basal fronto-orbital cortices and the medial portion of the temporal pole with the medial orbito-frontal cortex and gyrus rectus.

Note that given the considerable overlap between the different UF subcomponents (Figure 2), one could not expect to dissect each UF subcomponent separately. But, as illustrated in Figure 6, extracting the streamlines of the different UF subcomponents from their most superficial to deepest positions allows relating them to the dissection results. The most superficial streamlines terminating in IFG and belonging to the dorso-lateral UF subcomponent (Figure 6A) correspond to the most superficial lateral dissected fibers (Figure 5A). Once removed, it exposed the large fan-shaped streamlines belonging to the dorsolateral UF subcomponent (Figure 6B) and the horizontal fan-shaped streamlines of the ventro-lateral UF subcomponent (Figure 6C), also observed in dissection in Figure 5B. Continuing deeper after removing both dorso-lateral and ventro-lateral UF streamlines (Figure 6D) revealed the hook-shaped streamlines corresponding to the change of the orientation and the location of fibers observed during dissection. The most medial UF streamlines of the short antero-medial and postero-medial UF subcomponents correspond to the deepest and shortest UF dissected fibers (Figure 6E to 6G).

\section{Location of subcomponents within the UF stem}

The mean and individual center of mass coordinates of the subcomponents within the stem are shown in MNI space (Figure 7). They are situated at the ventral portion of the external and extreme capsule complex. Even with the large overlap between the 
subcomponents when looking at individuals, there is a clear topological organization of the subcomponents. Within the stem, the dorso-lateral UF subcomponent is located the most superiorly and laterally ( $C 1$ vs. $C 2 x$-coordinate: $p<.0001$ and $p=0.027$ in the left and right hemispheres, respectively, and z-coordinate: $p<.0001$ bilaterally), The ventro-lateral, ventromedial and short postero-medial UF subcomponents are arranged in a supero-inferior manner (see coronal slice, C2 vs. C3 z-coordinate: $p<.0001$ bilaterally, C3 vs. C4 zcoordinate: $p<.0001$ bilaterally) and the short antero-medial UF subcomponent is situated the most medially (C5 vs. C4 $x$-coordinate: $p=0.0017$ and 0.0002 in the left and right hemispheres, respectively). The mean coordinates of each UF subcomponent within the UF stem are given in Table 4.

\section{Discussion}

In this study, we combined an in vivo stem-based segmentation approach on an advanced anatomically constrained tractography with a post-mortem layer-by-layer brain blunt microdissection for evaluating the structural organization and terminations of the uncinate fasciculus.

We first observed a stretched UF pattern closely similar to the one that Dejerine's and others previously described (Dejerine and Dejerine-Klumpke, 1895; Gordinier, 1899; Testut, 1900) namely, with fibers that exhibit the classic hook shape connecting the frontal and anterior temporal lobes and, progressing posteriorly along the tract as they fan out, fibers that follow a straight horizontal trajectory and even curve somewhat in the inverse direction of the hook (Figure 8). The horizontal UF streamlines were dissociated from the IFOF lying adjacent to it at the stem level but separated by a gap in their posterior temporal course (Figure $1 \mathrm{E}$ and $5 \mathrm{E}$ ). The progressive posterior fanning of the UF transitioning into a horizontally-oriented trajectory can also be clearly seen in other dissection studies (Meynert, 1885; Kier et al., 2004), in particular Curran's dissection of the IFOF which demonstrated a 
relatively clear boundary between the IFOF and UF at the stem level and the inverse curvature of the most posterior UF fibers (Figure 8) (Curran, 1909). The difficulty involved in dissecting the posterior segment of the UF, which crosses with the inferior longitudinal fascicle (ILF) likely contributed to the neglect of these most posterior temporal uncinate fibers. Our microdissection of the ventral third of the external and extreme capsules confirmed a wide distribution of the uncinate fibers connecting the whole frontal lobe with the anterior temporal lobe (Figure 5). Starting from the original definition of Dejerine we performed a layer-by-layer dissection demonstrating both hook-shaped and fan-shaped distribution of UF terminations in the SFG, MFG, IFG and fronto-orbital and basal cortices and STG, MTG and temporal pole. As consequence of this wide and differently oriented distribution of terminations we demonstrated a progressive latero-medial and anterior torsion of the fibers of UF stem.

Then, we identified five subcomponents of the UF with distinct patterns of trajectory and cortical connections both in tractography (Figures 2-4) and microdissection (Figure 5). As mentioned in the introduction, the evidence for a multicomponent UF has been parsimoniously accumulating. Some early (Gordinier, 1899; Testut, 1900) and more recent (Ebeling and von Cramon, 1992) descriptions of the UF included subcomponent divisions of the bundle but no study has proposed a comprehensive and detailed account of its subcomponents. The present dorso-lateral UF subcomponent would correspond to that early observed by Gordinier (1899) and Testut (1900). In their Klingler's UF dissection study, Ebeling and Cramon's defined a ventral subcomponent consisting of connections between the gyrus rectus and fronto-subcallosal areas with temporal medial areas. It corresponds to the present short antero-medial UF subcomponent (Figure 3-C5) passing the most medially through the UF stem (Figure 7). Their dorsal subcomponent was limited to the orbito-frontal and inferior frontal terminations with the superior and middle temporal gyri, which rather corresponds to the present ventro-lateral UF subcomponent. Following the UF definition of Ebeling and Cramon, a recent study combined diffusion spectrum imaging tractography and post-mortem microdissection to describe an UF segmentation depending on its middle frontal 
and inferior frontal terminations but restricted to the hook-shaped uncinate streamlines (Leng et al., 2016). We demonstrate with both in vivo tractography and post-mortem microdissection that, beyond this previous division, a more dorso-lateral subcomponent connecting lateral frontal cortices with the superior and middle temporal gyri belongs to the UF.

A frontal to middle temporal tract, namely the extreme capsule tract (also known as the extreme capsule fiber tract) which lies dorso-laterally adjacent to the UF stem has been identified in the macaque monkey with axonal tracing (Schmahmann \& Pandya, 2006). The existence of such a tract has been discussed in humans (Parker et al., 2005; Saur et al., 2008; Weiller et al., 2011). There is however some ambiguity regarding its anatomical identity. It has been considered as an independent tract (Schmahmann and Pandya, 2006; Saur et al., 2008; Makris and Pandya, 2009), as the temporal part of the IFOF (Binney et al., 2012; Thiebaut de Schotten et al., 2012; Gierhan, 2013) and as part of the UF (Dejerine and Dejerine-Klumpke, 1895; Wernicke, 1908; Parker et al., 2005). Based on the present results the so-called extreme capsule fascicle may correspond to the most dorso-lateral subcomponent of the UF that precisely connects frontal to middle temporal cortices.

We also demonstrate a topographical organization of the subcomponents at the level of the UF stem (Figure 7) consistent with the subcomponents revealed in the layer-by-layer microdissection that progresses laterally to medially. The dorso-lateral UF subcomponent was systematically located superiorly and laterally with respect to the other subcomponents within the stem and the lateral and medial ventral UF subcomponents were organized in a supero-inferior manner along the central portion of the stem. The short anterior ventro-medial subcomponent passed through the stem at its most medial part. The post-mortem data also showed that the superficial wide and fan-shaped layers occupy the dorsal portion of the stem adjacent to the IFOF fibers while the deeper layers provide the shortest connections. This clustered organization as opposed to dispersed intermingling at the stem lends further support for the subcomponents as distinct sub-groups of the UF bundle. 


\section{Multifunctional roles for a multicomponent bundle}

Indeed due to the UF being a multicomponent bundle, its functional role has been difficult to disentangle. For instance, there have been conflicting results regarding whether or not lesions to the UF disrupt language function (Duffau et al., 2009; Papagno et al., 2011). Over a century ago Wernicke described the UF as one of "two important association bundles which must be considered in the anatomy of the speech regions" (Wernicke, 1908, p. 314315). More recently, the dorsal UF subcomponent has been postulated as a ventral language pathway responsible for semantic processing (Duffau et al., 2005; Saur et al., 2008). It has also been linked to associative learning (Von Der Heide et al., 2013; Thomas et al., 2015). The ventral subcomponents may have an important role in mnemonic associations and value judgments (Von Der Heide et al., 2013). A recent study investigating the circuitry of the temporal lobe suggested that the medial and lateral branches of the ventral UF may have distinct functions (Binney et al., 2012). They hypothesized that a ventro-lateral prefrontal branch might be related to social cognition and an orbital and medial frontal branch related to emotional valence.

Interestingly, we observed opposite asymmetries in two UF subcomponents, which clarifies the controversy surrounding the asymmetry of the UF. Previous studies on the asymmetry of the UF have only been conducted on the entire bundle and have been inconclusive with some revealing leftward (Kubicki et al., 2002; Hasan et al., 2009; Thomas et al., 2015; Leng et al., 2016), rightward (Highley et al., 2002; Rodrigo et al., 2007; Hau et al., 2016) and no lateralization (Thiebaut de Schotten et al., 2011b; Lebel et al., 2012). A previous diffusion imaging study observed a leftward asymmetry in voxels located at the superior portion and a rightward asymmetry in the inferior portion of the UF stem (Park et al., 2004) consistent with the present asymmetries of the UF subcomponents with the left lateralized dorso-lateral and right lateralized ventro-medial UF subcomponents, respectively. Tract quantitative measures (such as FA, number of streamlines, volume) depend on how the tract is segmented and therefore are contingent on its definition. Whether the dorsolateral subcomponent of the UF is considered or if only the "hook-shaped" portion is 
considered will affect the results on its asymmetry. The inconsistency in the accurate identification and localization of tracts has already been raised as an issue for comparing quantitative measures across groups and across studies (Jones, 2008). The quality of the data and the tractography results will also influence it. For example, the posterior part of the UF will be more difficult to obtain in DTI studies due to the crossing with the dominant ILF. This was a limitation of our previous study that did not allow observing the dorso-lateral UF subcomponent and a fortiori its leftward asymmetry. This underlines the need to study the UF in terms of its subcomponents instead of considering it as a homogeneous bundle whose definition has some ambiguities. The distinctive UF subcomponent asymmetries may be of interest for clinicians investigating patients with disorders that have been linked with the UF such as schizophrenia (Park et al., 2004; Price et al., 2008), clinical depression (Steffens et al., 2011; Zhang et al., 2012), Alzheimer's disease (Yasmin et al., 2008; Taoka et al., 2009) and psychopathy (Craig et al., 2009; Sobhani et al., 2015).

\section{Limitations}

Although the present stem-based approach allow a detailed and specific UF extraction, one may not exclude that some streamlines belonging to the adjacent IFOF bundle can be encompassed with the UF stem. Note first that the drawn "UF stem" ROI is made of $1-\mathrm{mm}^{3}$ voxels that encompass at best the stem structure without perfectly follow its more rounded aspects, then potentially catching some adjacent IFOF streamlines. These two bundles are indeed positioned side-by-side along the ventral external capsule to constitute the association pathways that connects the frontal cortex with the temporal, parietal and occipital cortices. We recently described the cortical termination of these two bundles by separating their respective stems (Hau et al. (2016)). We also showed that both UF and IFOF are projecting in the same frontal regions (MFG, IFG, LFOG, MFOG; see Figure 1B in Hau et al. (2016)). It is therefore not surprising that at the millimeter scale at which the whole tractogram was computed some intricated IFOF fibers were part of the UF stem and required their removal by placing a specific coronal slice filter (see Methods). 
In a more general point of view, we cannot rule out that some false-positive streamlines/fibers were identified with diffusion tractography and Klingler dissection. One may first emphasize that the main limitation of the blunt dissection, specially if performed by expert anatomists, is mostly related to false-negative results. In fact, over the last 10 years, studying the human white matter anatomy with Klingler dissection provided new insights about the structural connectivity of different associative, projection and commissural pathways that were contextually or separately confirmed by diffusion neuroimaging and tractography (Fernandez-Miranda et al., 2008; Thiebaut de Schotten et al., 2011a; Fernandez-Miranda et al., 2012; Sarubbo et al., 2013; De Benedictis et al., 2014; FernándezMiranda et al., 2015; De Benedictis et al., 2016; Innocenti et al., 2016; Sarubbo et al., 2016; Wang et al., 2016).

Considering the dissection tractography, we performed a stem-based segmentation approach with an advanced anatomically constrained tractography, which minimized the known limitations of diffusion tractography (Jones et al., 2013; Jbabdi et al., 2015). We first addressed the problem of crossing/kissing streamline configurations by using a CSD tractography (Descoteaux et al., 2009; Tournier et al., 2012). Furthermore, the particle filtering of tractography algorithm with anatomical prior enabled us to improve tracking results, by reducing premature stopping through areas of low anisotropy (Girard et al., 2014) and better following fan-shaped UF fibers up to their cortical termination as recently described for both UF and IFOF (Hau et al., 2016) and the anterior part of the frontal corpus callosum (De Benedictis et al., 2016).

One may criticize that the Klingler dissection has a low spatial resolution as compared to other post-mortem anatomical techniques (e.g. histology or polarized-ligth imaging), but it has the same resolution as diffusion tractography of the order of the millimeter and it provides direct visualization of the fibers without losing their tridimensional coherence. Interestingly, a recent study demonstrated that the freezing-defrosting of the brain during the Klingler preparation creates extra-axonal lacunas that facilitates the separation of the fibers during the dissection but without destroying the axonal structure (Zemmoura et al., 2016). 
These results first confirm why detailed fiber dissection is possible using Klingler's method, but also explain why studying fiber crossings during dissection is problematic, as the anatomist has to cut the crossing fibers to continue following a bundle of interest. This might be rather considered as an advantage since Klingler dissection can efficiently track one specific bundle through a crossing region. We recently demonstrated that the callosal fibers can be dissected in the frontal cortex up to their cortical termination by performing a layer-bylayer dissection through the crisscross of the association pathways (Sarubbo et al., 2016). In the present study, once the UF fibers exposed around the anterior and ventral third of the external and extreme capsules, we were able to dissect the complete UF fibers with a progressive removal of the white matter layer-by-layer through the crisscross of the projections fibers passing through the anterior arm of the internal capsule, the callosal fibers and some adjacent association patways (SLF, AF, IFOF). Despite the unique chance to follow a specific bundle starting from the dense core (i.e. the stem) up to the cortical terminations (according to the cortex sparing technique applied in this study), the blunt microdissection did not provide quantitative data regarding these terminations nor additional information about further cortical territories of terminations (i.e. false-negatives) or the full pattern of reciprocal connectivity encompassed by UF fibers. But combining in vivo anatomically constrained diffusion tractography with post-mortem Klingler dissection appreciably reduces these limitations to more reliably describe the white matter pathways.

\section{Conclusion}

In the present study we delineated the human uncinate fasciculus and inferred its subcomponent organization with extended cortical connections within the frontal and temporal cortices using advanced anatomical constrained particle-filtering tractography in vivo and post-mortem microdissection. Several other white matter pathways have recently been identified as multicomponent bundles including the IFOF (Martino et al., 2010; Sarubbo 
et al., 2013; Hau et al., 2016), the SLF/AF (Catani et al., 2005; Makris et al., 2005; Wang et al., 2016) suggesting that our knowledge has much to gain regarding the detailed anatomy of the fiber pathways. Shifting our understanding of the UF to a multicomponent bundle will improve the specificity of studies relating it to function and disorders and will be helpful for surgical application. 


\section{Figure legends}

Figure 1. Stem-based anatomical virtual dissection of UF. A. View of $10 \%$ of the left intrahemispheric streamlines of a single whole-brain tractogram; B to C. View of $10 \%$ of the streamlines with one termination in the frontal lobe (in orange) and the other either in the temporal, cingulate, parietal or occipital lobes (in blue); C (zoom in). Initial rectangular ROI (in red) drawn at the level of the hook-shaped streamlines (yellow arrow); D. View of $100 \%$ of the streamlines once the UF stem size adjusted (in red); E. All the streamlines passing through the UF stem are exposed. An additional coronal slice filter (red frame) was used to remove the remaining IFOF streamlines. F. Application of the outlier removal algorithm to prune outlier streamlines $(\mathbf{G})$ and provides the final UF bundle $(\mathbf{H})$.

Figure 2. Visualization of the clusters in three-dimensional space obtained from the entire dataset $(n=30)$. On the left, the clusters are represented as tube renderings showing the course and terminations of each cluster group. On the right, $5 \%$ of the total of streamlines corresponding to each cluster on the group data (both left and right hemispheres) are shown.

Figure 3. The five UF subcomponents, subsampled at $20 \%$ of the total of streamlines for each cluster on the group data for visual display.

Figure 4. Mean connectional anatomies of each subcomponent. The lines thickness is proportional to the mean normalized number of streamlines connecting a pair of regions. SFG, MFG, IFG: superior, middle and inferior frontal gyri; LFOG, MFOG: lateral and medial fronto-orbital gyri; RG: rectus gyrus; STG, MTG, ITG: superior, middle and inferior temporal gyri; Tpole: temporal pole; FuG: fusiform gyrus. 
Figure 5. A. View of the fronto-temporal opercular region with the exposed UF stem exposing the fibers (posterior green line) connecting the middle third of the STG and the anterior third of the MTG (inferior green rectangle) with the pars opercularis and triangularis of the IFG (superior green rectangle); the fibers (anterior green lines) connecting pars orbitalis and basal fronto-orbital cortex (inferior green circle) with the basal fronto-orbital cortex (superior green circle). B. Complete view of a left hemisphere with the fibers of the second layer. The posterior portion (posterior red line) connect the middle third of STG and middle/anterior third of MTG (inferior red rectangle) with the posterior third of SFG and MFG (superior red rectangle). The anterior portion (anterior red line) connects the antero-lateral portion of the temporal pole (inferior red circle) with the dorsal portion of the anterior third of IFG (superior red circle). C. Detail of the anterior temporal and frontal cortices to demonstrate the fibers connecting: the lateral temporal pole (inferior red circle) with the lateral fronto-orbital cortices (superior red circle); the anterior temporal pole (inferior green circle) with the latero-fronto orbital cortices (superior green circle). D. Detail of the anterior portion of the frontal and temporal poles showing the deepest layers. The yellow line follows the course of the most lateral and dorsal fibers connecting the antero-medial temporal pole (inferior yellow circle) with the basal fronto-orbital cortices (superior yellow circle). The blue and orange line highlights the course of the deepest short UF fibers connecting the medial temporal pole (inferior blue and orange circles) with the medial orbito-frontal cortex (superior blue circle) and gyrus rectus (superior orange circle). E. Distinction between the UF and IFOF stems.

Figure 6. Latero-medial organization of the five UF subcomponents for the left hemisphere, subsampled at $20 \%$ of the total of streamlines for each cluster on the group data (see text for details).

Figure 7. Topological organization of the subcomponents within the UF stem. Mean and individual subject centre of mass locations of each subcomponent at the intersection with the 
delineated stem in $\mathrm{MNI}$ space projected on the mean coronal and axial slice. Colors correspond to each class $(\mathrm{C} 1=$ deep red, $\mathrm{C} 2=$ cyan, $\mathrm{C} 3=$ yellow, $\mathrm{C} 4=$ green, $\mathrm{C} 5=\mathrm{blue})$.

Figure 8. Dejerine's schematic representation of the UF at medial $(A)$ and lateral $(B)$ stages of dissection (Figures 366-367 from Dejerine \& Dejerine-Klumpke, 1895). Curran's dissection of the left (C) and right (D) IFOF where the dorsal horizontal subcomponent of the UF can clearly be seen (Plate I from Curran, 1909).

\section{Supplementary material.}

Anatomical plates of the principal streamlines of the five subcomponents of the uncinate fascicle superimposed on coronal views of the MNI single-subject from $y=+55 \mathrm{~mm}$ to $y=-40$ mm. Plate 1: dorsal UF (in red); Plate 2: ventro-lateral UF (in green); Plate 3: ventro-medial UF (in yellow); Plate 4: short posterior ventro-medial UF (in cyan); Plate 5: short anterior ventro-medial UF (in orange). L: left; R: right. 


\section{References}

Axer $\mathrm{H}$, Klingner CM, Prescher A (2013) Fiber anatomy of dorsal and ventral language streams. Brain Lang 127:192-204.

Bajada CJ, Lambon Ralph MA, Cloutman LL (2015) Transport for language south of the Sylvian fissure: The routes and history of the main tracts and stations in the ventral language network. Cortex 69:141-151.

Binney RJ, Parker GJ, Lambon Ralph MA (2012) Convergent connectivity and graded specialization in the rostral human temporal lobe as revealed by diffusion-weighted imaging probabilistic tractography. J Cogn Neurosci 24:1998-2014.

Catani M, Jones DK, Ffytche DH (2005) Perisylvian language networks of the human brain. Annals of Neurology 57:8-16.

Catani M, Howard RJ, Pajevic S, Jones DK (2002) Virtual in Vivo Interactive Dissection of White Matter Fasciculi in the Human Brain. Neuroimage 17:77-94.

Côté M-A, Garyfallidis E, Larochelle H, Descoteaux M (2015) Cleaning up the mess: tractography outlier removal using hierarchical QuickBundles clustering. In: 23rd ISMRM Annual Meeting. Toronto, Canada.

Craig MC, Catani M, Deeley Q, Latham R, Daly E, Kanaan R, Picchioni M, McGuire PK, Fahy T, Murphy DG (2009) Altered connections on the road to psychopathy. Mol Psychiatry 14:946-953, 907.

Curran EJ (1909) A new association fiber tract in the cerebrum with remarks on the fiber tract dissection method of studying the brain. Journal of Comparative Neurology and Psychology 19:645-656.

De Benedictis A, Petit L, Descoteaux M, Marras CE, Barbareschi M, Corsini F, Dallabona M, Chioffi F, Sarubbo $S$ (2016) New insights in the homotopic and heterotopic connectivity of the frontal part of the human corpus callosum revealed by microdissection and diffusion tractography. Hum Brain Mapp in press.

De Benedictis A, Duffau H, Paradiso B, Grandi E, Balbi S, Granieri E, Colarusso E, Chioffi F, Marras CE, Sarubbo S (2014) Anatomo-functional study of the temporo-parieto-occipital region: dissection, tractographic and brain mapping evidence from a neurosurgical perspective. J Anat 225:132-151.

Dejerine J, Dejerine-Klumpke A (1895) Anatomie des centres nerveux. Tome 1. Paris: Rueff et Cie.

Dempster AP, Laird NM, Rubin DB (1976) Maximum likelihood from incomplete data via the EM algorithm. Journal of the Royal Statistical Society Series B (Methodological) 39.

Descoteaux M, Deriche R, Knosche TR, Anwander A (2009) Deterministic and probabilistic tractography based on complex fibre orientation distributions. IEEE Trans Med Imaging 28:269-286.

Duffau H, Gatignol P, Moritz-Gasser S, Mandonnet E (2009) Is the left uncinate fasciculus essential for language? A cerebral stimulation study. J Neurol 256:382-389.

Duffau H, Gatignol P, Mandonnet E, Peruzzi P, Tzourio-Mazoyer N, Capelle L (2005) New insights into the anatomo-functional connectivity of the semantic system: a study using corticosubcortical electrostimulations. Brain 128:797-810.

Ebeling U, von Cramon D (1992) Topography of the uncinate fascicle and adjacent temporal fiber tracts. Acta Neurochir (Wien) 115:143-148.

Fernández-Miranda J, Wang Y, Pathak S, Stefaneau L, Verstynen T, Yeh F-C (2015) Asymmetry, connectivity, and segmentation of the arcuate fascicle in the human brain. Brain Structure and Function 220:1665-1680.

Fernandez-Miranda JC, Rhoton AL, Kakizawa Y, Choi C, Alvarez-Linera J (2008) The claustrum and its projection system in the human brain: a microsurgical and tractographic anatomical study. Journal of Neurosurgery 108:764-774. 
Fernandez-Miranda JC, Pathak S, Engh J, Jarbo K, Verstynen T, Yeh FC, Wang Y, Mintz A, Boada F, Schneider W, Friedlander R (2012) High-definition fiber tractography of the human brain: neuroanatomical validation and neurosurgical applications. Neurosurgery 71:430-453.

Forkel SJ, Thiebaut de Schotten M, Kawadler JM, Dell'Acqua F, Danek A, Catani M (2014) The anatomy of fronto-occipital connections from early blunt dissections to contemporary tractography. Cortex 56:73-84.

Garyfallidis E, Brett M, Correia MM, Williams GB, Nimmo-Smith I (2012) QuickBundles, a method for tractography simplification. Frontiers in Neuroscience 6.

Gierhan SME (2013) Connections for auditory language in the human brain. Brain and Language 127:205-221.

Girard G, Whittingstall K, Deriche R, Descoteaux M (2014) Towars quantitative connectivity analysis: Reducing tractography biaises. Neurolmage 98:266-278.

Gordinier HC (1899) The gross and minute anatomy of the central nervous system. Philadelphia (USA): Blakiston's Son \& Co.

Hasan KM, Iftikhar A, Kamali A, Kramer LA, Ashtari M, Cirino PT, Papanicolaou AC, Fletcher JM, Ewing-Cobbs $L$ (2009) Development and aging of the healthy human brain uncinate fasciculus across the lifespan using diffusion tensor tractography. Brain research 1276:67-76.

Hau J, Sarubbo S, Perchey G, Crivello F, Zago L, Mellet E, Jobard G, Joliot M, Mazoyer B, TzourioMazoyer N, Petit $L$ (2016) Cortical terminations of the inferior fronto-occipital and uncinate fasciculi: Stem-based anatomical virtual dissection Frontiers in Neuroanatomy 10:58.

Hernando KA, Szaflarski JP, Ver Hoef LW, Lee S, Allendorfer JB (2015) Uncinate fasciculus connectivity in patients with psychogenic nonepileptic seizures: A preliminary diffusion tensor tractography study. Epilepsy Behav 45:68-73.

Highley JR, Walker MA, Esiri MM, Crow TJ, Harrison PJ (2002) Asymmetry of the Uncinate Fasciculus: A Post-mortem Study of Normal Subjects and Patients with Schizophrenia. Cerebral Cortex 12:1218-1224.

Innocenti GM, Dyrby TB, Andersen KW, Rouiller EM, Caminiti R (2016) The Crossed Projection to the Striatum in Two Species of Monkey and in Humans: Behavioral and Evolutionary Significance. Cereb Cortex.

Jbabdi S, Sotiropoulos SN, Haber SN, Van Essen DC, Behrens TE (2015) Measuring macroscopic brain connections in vivo. Nat Neurosci 18:1546-1555.

Jones DK (2008) Studying connections in the living human brain with diffusion MRI. Cortex 44:936952.

Jones DK, Knosche TR, Turner R (2013) White matter integrity, fiber count, and other fallacies: the do's and don'ts of diffusion MRI. Neuroimage 73:239-254.

Kier EL, Staib LH, Davis LM, Bronen RA (2004) MR Imaging of the Temporal Stem: Anatomic Dissection Tractography of the Uncinate Fasciculus, Inferior Occipitofrontal Fasciculus, and Meyer's Loop of the Optic Radiation. American Journal of Neuroradiology 25:677-691.

Kubicki M, Westin CF, Maier SE, Frumin M, Nestor PG, Salisbury DF, Kikinis R, Jolesz FA, McCarley RW, Shenton ME (2002) Uncinate fasciculus findings in schizophrenia: a magnetic resonance diffusion tensor imaging study. Am J Psychiatry 159:813-820.

Lebel C, Gee M, Camicioli R, Wieler M, Martin W, Beaulieu C (2012) Diffusion tensor imaging of white matter tract evolution over the lifespan. Neurolmage 60:340-352.

Leng B, Han S, Bao Y, Zhang H, Wang Y, Wu Y, Wang Y (2016) The uncinate fasciculus as observed using diffusion spectrum imaging in the human brain. Neuroradiology.

Makris N, Pandya D (2009) The extreme capsule in humans and rethinking of the language circuitry. Brain Structure and Function 213:343-358.

Makris N, Kennedy DN, Mclnerney S, Sorensen AG, Wang R, Caviness VS, Pandya DN (2005) Segmentation of Subcomponents within the Superior Longitudinal Fascicle in Humans: A Quantitative, In Vivo, DT-MRI Study. Cerebral Cortex 15:854-869.

Mars RB, Foxley S, Verhagen L, Jbabdi S, Sallet J, Noonan MP, Neubert FX, Andersson JL, Croxson PL, Dunbar RI, Khrapitchev AA, Sibson NR, Miller KL, Rushworth MF (2015) The extreme capsule 
fiber complex in humans and macaque monkeys: a comparative diffusion MRI tractography study. Brain Struct Funct.

Martino J, Vergani F, Robles SG, Duffau H (2010) New insights into the anatomic dissection of the temporal stem with special emphasis on the inferior fronto-occipital fasciculus: implications in surgical approach to left mesiotemporal and temporoinsular structures. Neurosurgery 66:4-12.

Martino J, De Witt Hamer PC, Vergani F, Brogna C, de Lucas EM, Vázquez-Barquero A, García-Porrero JA, Duffau H (2011) Cortex-sparing fiber dissection: an improved method for the study of white matter anatomy in the human brain. Journal of Anatomy 219:531-541.

Mazoyer B, Mellet E, Perchey G, Zago L, Crivello F, Jobard G, Delcroix N, Vigneau M, Leroux G, Petit L, Joliot M, Tzourio-Mazoyer N (2016) BIL\&GIN: A neuroimaging, cognitive, behavioral, and genetic database for the study of human brain lateralization. Neuroimage 124 Part B:12251231.

Meola A, Comert A, Yeh FC, Stefaneanu L, Fernandez-Miranda JC (2015) The controversial existence of the human superior fronto-occipital fasciculus: Connectome-based tractographic study with microdissection validation. Hum Brain Mapp 36:4964-4971.

Meynert T (1885) Psychiatry: Clinical Treatise on the Diseases of the Fore-Brain, trans. B. Sachs. New York \& London: GP Putnam.

Oishi K, Faria AV, Hsu J, Tippett D, Mori S, Hillis AE (2015) Critical role of the right uncinate fasciculus in emotional empathy. Ann Neurol 77:68-74.

Oishi K, Faria A, Jiang H, Li X, Akhter K, Zhang J, Hsu JT, Miller MI, van Zijl PCM, Albert M, Lyketsos CG, Woods R, Toga AW, Pike GB, Rosa-Neto P, Evans A, Mazziotta J, Mori S (2009) Atlas-based whole brain white matter analysis using large deformation diffeomorphic metric mapping: Application to normal elderly and Alzheimer's disease participants. Neurolmage 46:486-499.

Papagno C, Miracapillo C, Casarotti A, Romero Lauro LJ, Castellano A, Falini A, Casaceli G, Fava E, Bello $L$ (2011) What is the role of the uncinate fasciculus? Surgical removal and proper name retrieval. Brain 134:405-414.

Park H-J, Westin C-F, Kubicki M, Maier SE, Niznikiewicz M, Baer A, Frumin M, Kikinis R, Jolesz FA, McCarley RW, Shenton ME (2004) White matter hemisphere asymmetries in healthy subjects and in schizophrenia: a diffusion tensor MRI study. Neurolmage 23:213-223.

Parker GJM, Luzzi S, Alexander DC, Wheeler-Kingshott CAM, Ciccarelli O, Lambon Ralph MA (2005) Lateralization of ventral and dorsal auditory-language pathways in the human brain. Neurolmage 24:656-666.

Phan KL, Orlichenko A, Boyd E, Angstadt M, Coccaro EF, Liberzon I, Arfanakis K (2009) Preliminary evidence of white matter abnormality in the uncinate fasciculus in generalized social anxiety disorder. Biol Psychiatry 66:691-694.

Price G, Cercignani M, Parker GJ, Altmann DR, Barnes TR, Barker GJ, Joyce EM, Ron MA (2008) White matter tracts in first-episode psychosis: a DTI tractography study of the uncinate fasciculus. Neuroimage 39:949-955.

Rodrigo S, Oppenheim C, Chassoux F, Golestani N, Cointepas Y, Poupon C, Semah F, Mangin JF, Le Bihan D, Meder JF (2007) Uncinate fasciculus fiber tracking in mesial temporal lobe epilepsy. Initial findings. Eur Radiol 17:1663-1668.

Sarubbo S, De Benedictis A, Maldonado IL, Basso G, Duffau H (2013) Frontal terminations for the inferior fronto-occipital fascicle: anatomical dissection, DTI study and functional considerations on a multi-component bundle. Brain structure \& function 218:21-37.

Sarubbo S, De Benedictis A, Merler S, Mandonnet E, Barbareschi M, Dallabona M, Chioffi F, Duffau H (2016) Structural and functional integration between dorsal and ventral language streams as revealed by blunt dissection and direct electrical stimulation. Hum Brain Mapp in press.

Sarubbo S, De Benedictis A, Milani P, Paradiso B, Barbareschi M, Rozzanigo U, Colarusso E, Tugnoli V, Farneti M, Granieri E, Duffau H, Chioffi F (2015) The course and the anatomo-functional relationships of the optic radiation: a combined study with 'post mortem' dissections and 'in vivo' direct electrical mapping. J Anat 226:47-59. 
Saur D, Kreher BW, Schnell S, Kummerer D, Kellmeyer P, Vry MS, Umarova R, Musso M, Glauche V, Abel S, Huber W, Rijntjes M, Hennig J, Weiller C (2008) Ventral and dorsal pathways for language. Proc Natl Acad Sci U S A.

Schmahmann JD, Pandya DN (2006) Fiber pathways of the brain. New York: Oxford University Press.

Schwartz G (1978) Estimating the dimension of a model. The annals of statistics 6:461-464.

Sobhani M, Baker L, Martins B, Tuvblad C, Aziz-Zadeh L (2015) Psychopathic traits modulate microstructural integrity of right uncinate fasciculus in a community population. Neuroimage Clin 8:32-38.

Steffens DC, Taylor WD, Denny KL, Bergman SR, Wang L (2011) Structural integrity of the uncinate fasciculus and resting state functional connectivity of the ventral prefrontal cortex in late life depression. PLoS One 6:e22697.

Taoka T, Morikawa M, Akashi T, Miyasaka T, Nakagawa H, Kiuchi K, Kishimoto T, Kichikawa K (2009) Fractional anisotropy--threshold dependence in tract-based diffusion tensor analysis: evaluation of the uncinate fasciculus in Alzheimer disease. AJNR Am J Neuroradiol 30:17001703.

Testut L (1900) Traité d'anatomie humaine. Tome 2. Paris: Octave Doin.

Thiebaut de Schotten M, Dell'Acqua F, Valabregue R, Catani M (2012) Monkey to human comparative anatomy of the frontal lobe association tracts. Cortex 48:82-96.

Thiebaut de Schotten M, Dell'Acqua F, Forkel SJ, Simmons A, Vergani F, Murphy DGM, Catani M (2011a) A lateralized brain network for visuospatial attention. Nat Neurosci 14:1245-1246.

Thiebaut de Schotten M, Ffytche D, Bizzi A, Dell'acqua F, Allin M, Walshe M, Murray R, Williams S, Murphy DGM, Catani M (2011b) Atlasing location, asymmetry and inter-subject variability of white matter tracts in the human brain with MR diffusion tractography. Neurolmage 54:4959.

Thomas C, Avram A, Pierpaoli C, Baker C (2015) Diffusion MRI properties of the human uncinate fasciculus correlate with the ability to learn visual associations. Cortex 72:65-78.

Tournier J-D, Calamante F, Connelly A, Tournier J-D, Calamante F, Connelly A (2012) MRtrix: Diffusion tractography in crossing fiber regions. Int J Imaging Syst Technol 22:53-66.

Tournier JD, Calamante F, Connelly A (2007) Robust determination of the fibre orientation distribution in diffusion MRI: Non-negativity constrained super-resolved spherical deconvolution. Neurolmage 35:1459-1472.

Travers N (2008) L'étude micro-anatomique des fibres du faisceau unciné et ses implications dans la chirurgie fronto-temporo-insulaire. Académie Nationale de Chirurgie 7:31-41.

Ture U, Yasargil MG, Friedman AH, Al-Mefty O (2000) Fiber Dissection Technique: Lateral Aspect of the Brain. Neurosurgery 47:417-427.

Von Der Heide RJ, Skipper LM, Klobusicky E, Olson IR (2013) Dissecting the uncinate fasciculus: disorders, controversies and a hypothesis. Brain 136:1692-1707.

Wang X, Pathak S, Stefaneanu L, Yeh FC, Li S, Fernandez-Miranda JC (2016) Subcomponents and connectivity of the superior longitudinal fasciculus in the human brain. Brain Struct Funct 221:2075-2092.

Wassermann D, Makris N, Rathi Y, Shenton M, Kikinis R, Kubicki M, Westin CF (2013) On describing human white matter anatomy: the white matter query language. Medical image computing and computer-assisted intervention : MICCAI International Conference on Medical Image Computing and Computer-Assisted Intervention 16:647-654.

Weiller C, Bormann T, Saur D, Musso M, Rijntjes M (2011) How the ventral pathway got lost - And what its recovery might mean. Brain and Language 118:29-39.

Wernicke C (1908) Modern clinical medical diseases of the nervous system. New York: AppletonCentury-Crofts.

Wong NM, Cheung SH, Chan CC, Zeng H, Liu YP, So KF, Lee TM (2015) Diffusivity of the uncinate fasciculus in heroin users relates to their levels of anxiety. Transl Psychiatry 5:e554.

Yasmin H, Nakata Y, Aoki S, Abe O, Sato N, Nemoto K, Arima K, Furuta N, Uno M, Hirai S, Masutani Y, Ohtomo K (2008) Diffusion abnormalities of the uncinate fasciculus in Alzheimer's disease: 
diffusion tensor tract-specific analysis using a new method to measure the core of the tract. Neuroradiology 50:293-299.

Yeatman JD, Weiner KS, Pestilli F, Rokem A, Mezer A, Wandell BA (2014) The vertical occipital fasciculus: a century of controversy resolved by in vivo measurements. Proc Natl Acad Sci U S A 111:E5214-5223.

Zemmoura I, Blanchard E, Raynal PI, Rousselot-Denis C, Destrieux C, Velut S (2016) How Klingler's dissection permits exploration of brain structural connectivity? An electron microscopy study of human white matter. Brain Struct Funct 221:2477-2486.

Zhang A, Leow A, Ajilore O, Lamar M, Yang S, Joseph J, Medina J, Zhan L, Kumar A (2012) Quantitative tract-specific measures of uncinate and cingulum in major depression using diffusion tensor imaging. Neuropsychopharmacology 37:959-967. 

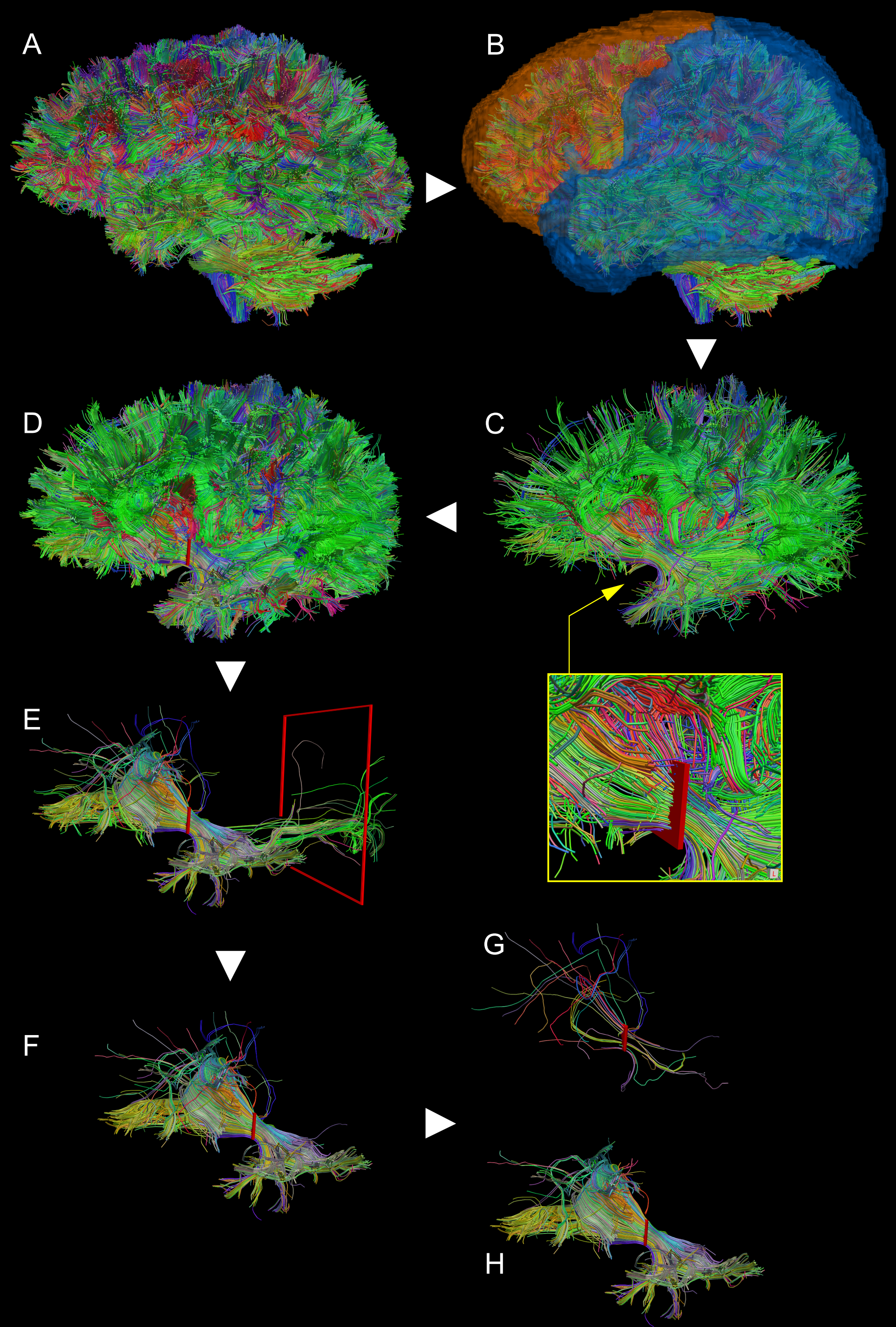

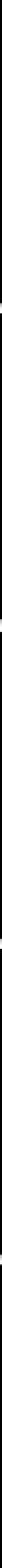
Left
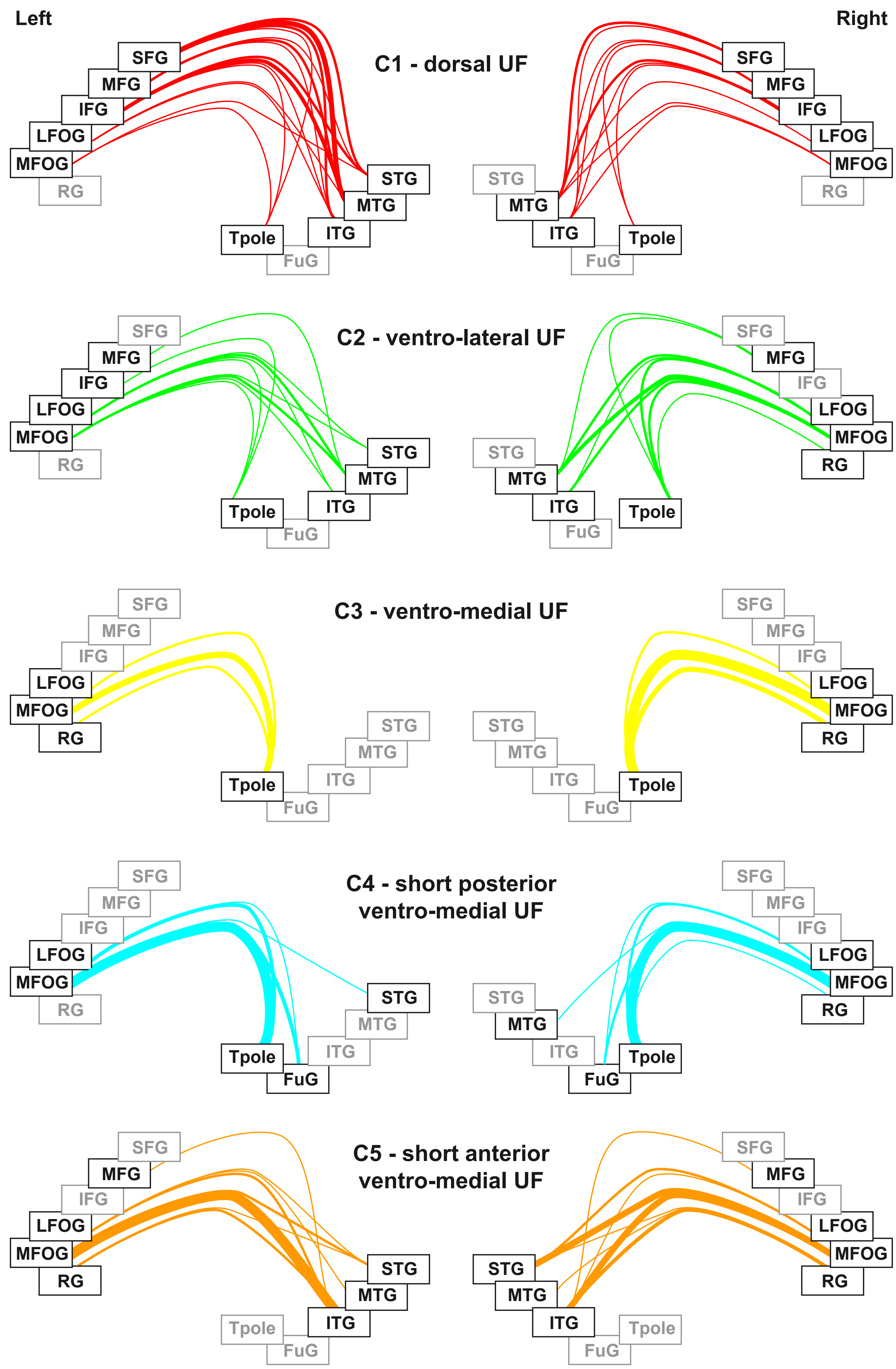

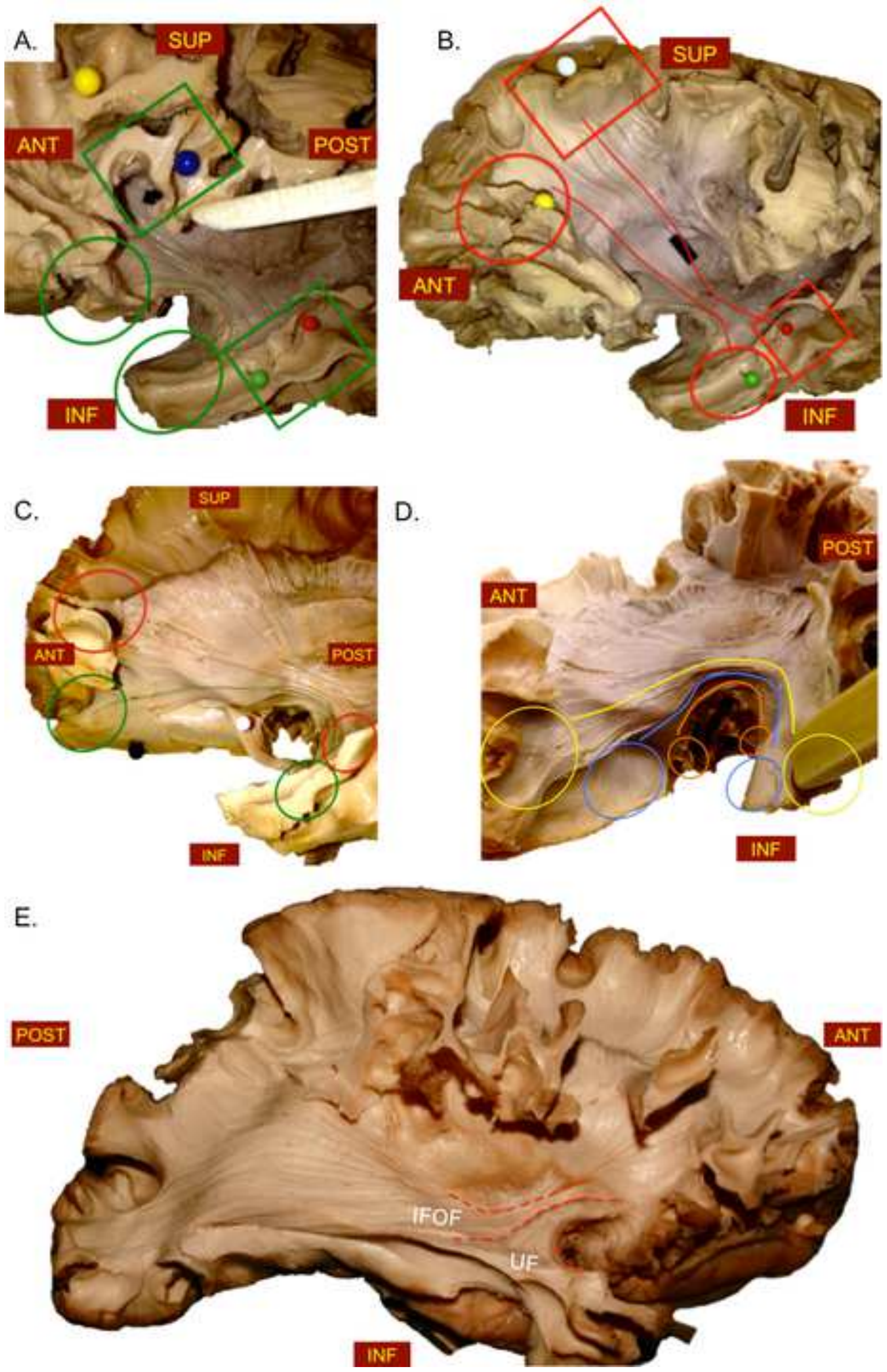
Table 1. A. Mean \pm standard deviation of the center of mass of the drawn UF stems in MNI space. B. Mean \pm standard deviation of the location of each subcomponent within the UF stem.

\begin{tabular}{rlllllll}
\hline & \multicolumn{3}{c}{ Left hemisphere } & \multicolumn{3}{c}{ Right hemisphere } \\
& $\mathbf{x}$ & $\mathbf{y}$ & $\mathbf{z}$ & $\mathbf{x}$ & $\mathbf{y}$ & $\mathbf{z}$ \\
UF stem & $-30 \pm 2$ & $2 \pm 3$ & $-10 \pm 1$ & $29 \pm 2$ & $5 \pm 3$ & $-10 \pm 1$ \\
\hline Dorso-lateral UF & $-31 \pm 1$ & $2 \pm 3$ & $-9 \pm 1$ & $30 \pm 1$ & $5 \pm 3$ & $-9 \pm 2$ \\
Ventro-lateral UF & $-29 \pm 2$ & $2 \pm 3$ & $-11 \pm 1$ & $29 \pm 1$ & $5 \pm 3$ & $-10 \pm 1$ \\
Ventro-medial UF & $-30 \pm 2$ & $2 \pm 3$ & $-12 \pm 1$ & $29 \pm 1$ & $5 \pm 3$ & $-11 \pm 1$ \\
Postero-medial UF & $-29 \pm 2$ & $1 \pm 3$ & $-13 \pm 1$ & $29 \pm 2$ & $5 \pm 3$ & $-12 \pm 1$ \\
Antero-medial UF & $-28 \pm 2$ & $2 \pm 3$ & $-12 \pm 1$ & $28 \pm 3$ & $5 \pm 3$ & $-10 \pm 1$ \\
\hline
\end{tabular}


Table. 2. UF subcomponents properties. Frequency is determined as the number of subjects with a number of subcomponent streamlines exceeding the threshold of $2 \%$ of the UF streamlines. Number, volume $(\mathrm{cm} 3)$ and length $(\mathrm{mm})$ of streamlines are expressed as the mean \pm standard deviation with this number of subjects.

\begin{tabular}{|c|c|c|c|c|c|c|c|c|c|c|}
\hline & \multicolumn{2}{|l|}{ C1 } & \multicolumn{2}{|l|}{ C2 } & \multicolumn{2}{|l|}{ C3 } & \multicolumn{2}{|l|}{ C4 } & \multicolumn{2}{|l|}{ C5 } \\
\hline & $\mathbf{L}$ & $\mathbf{R}$ & L & $\mathbf{R}$ & $\mathbf{L}$ & $\mathbf{R}$ & $\mathbf{L}$ & $\mathbf{R}$ & $\mathbf{L}$ & $\mathbf{R}$ \\
\hline Freq. & $27(90 \%)$ & $19(63 \%)$ & $30(100 \%)$ & $30(100 \%)$ & $26(87 \%)$ & $30(100 \%)$ & $29(97 \%)$ & $28(93 \%)$ & $30(100 \%)$ & $24(80 \%)$ \\
\hline Number & $276 \pm 200$ & $122 \pm 147$ & $593 \pm 296$ & $729 \pm 453$ & $256 \pm 231$ & $575 \pm 362$ & $273 \pm 169$ & $358 \pm 285$ & $191 \pm 111$ & $205 \pm 201$ \\
\hline Volume & $6.6 \pm 3.7$ & $3.6 \pm 2.8$ & $7.3 \pm 2.1$ & $8.4 \pm 2.6$ & $2.4 \pm 1.3$ & $3.8 \pm 1.4$ & $1.4 \pm 0.6$ & $1.6 \pm 0.7$ & $1.3 \pm 0.7$ & $1.8 \pm 1.2$ \\
\hline Length & $100.8 \pm 8.5$ & $96.4 \pm 12.6$ & $81.6 \pm 8.5$ & $81.1 \pm 8.4$ & $70.1 \pm 16.0$ & $72.6 \pm 6.5$ & $47.7 \pm 5.5$ & $40.1 \pm 9.2$ & $31.2 \pm 10.6$ & $46.1 \pm 10.2$ \\
\hline
\end{tabular}

Table 3. Mean asymmetry indexes of the number of streamlines and volume for the UF subcomponents (C1 to C5).

Significant asymmetry indices $(\mathrm{Al})$ are denoted with an asterisk $(\mathrm{p}<0.05)$.

\begin{tabular}{llllll}
\hline $\mathbf{n = 3 0}$ & C1 & C2 & C3 & C4 & C5 \\
\hline \# streamlines Al & -0.24 & 0.06 & $0.32^{*}$ & 0.10 & 0.00 \\
Tract volume Al & $-0.19^{*}$ & 0.07 & $0.19^{*}$ & 0.04 & $0.17^{*}$ \\
\hline
\end{tabular}


Anatomical plates with the principal streamlines of the five different subcomponents of the uncinate fascicle

dorsal UF

ventro-lateral UF

ventro-medial UF

short posterior ventro-medial UF

short anterior ventro-medial UF 
$+55$

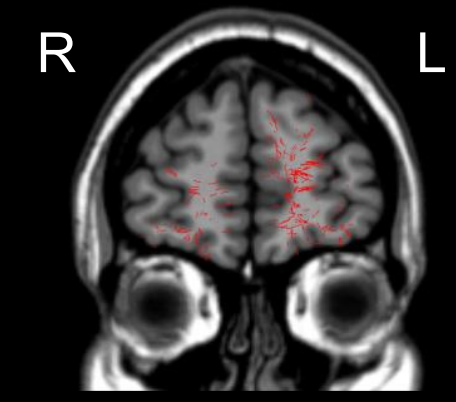

$+30$

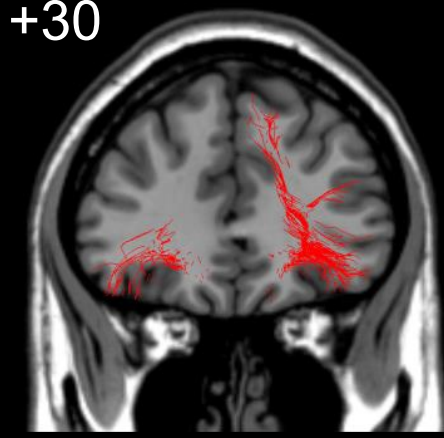

$+5$

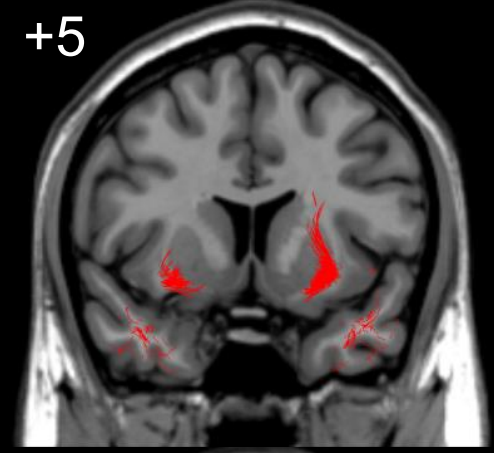

$-2$

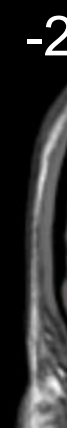

$+50$

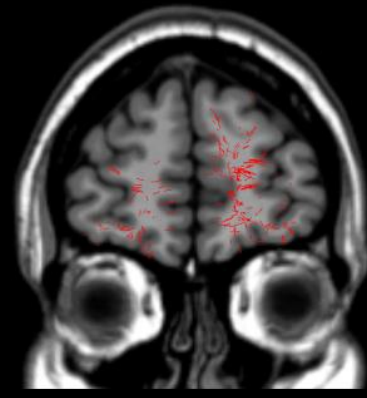

$+25$

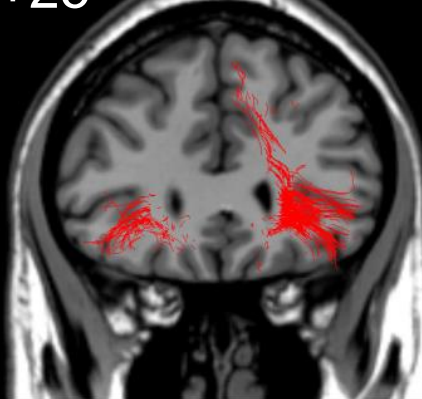

0
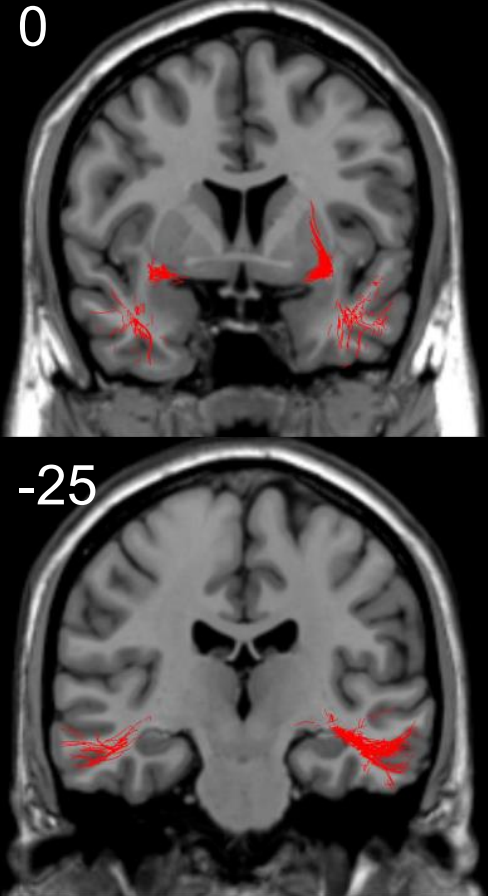

$+45$

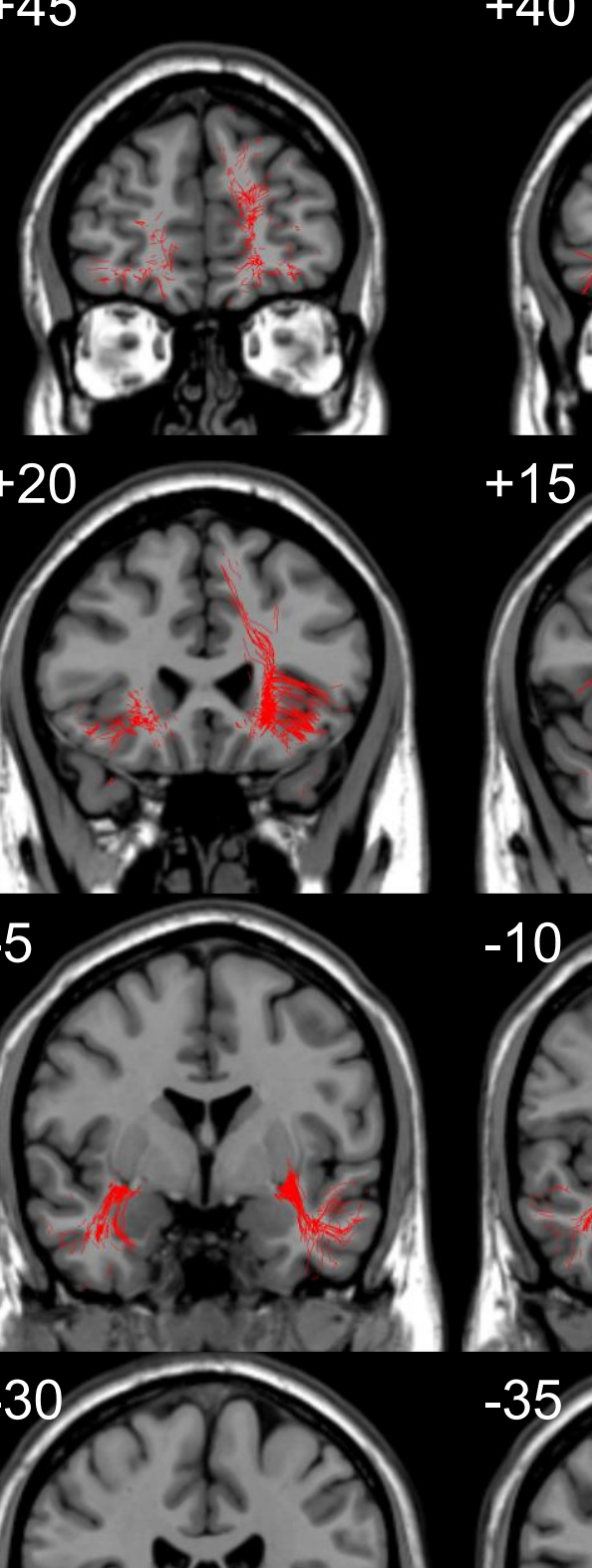

$+20$

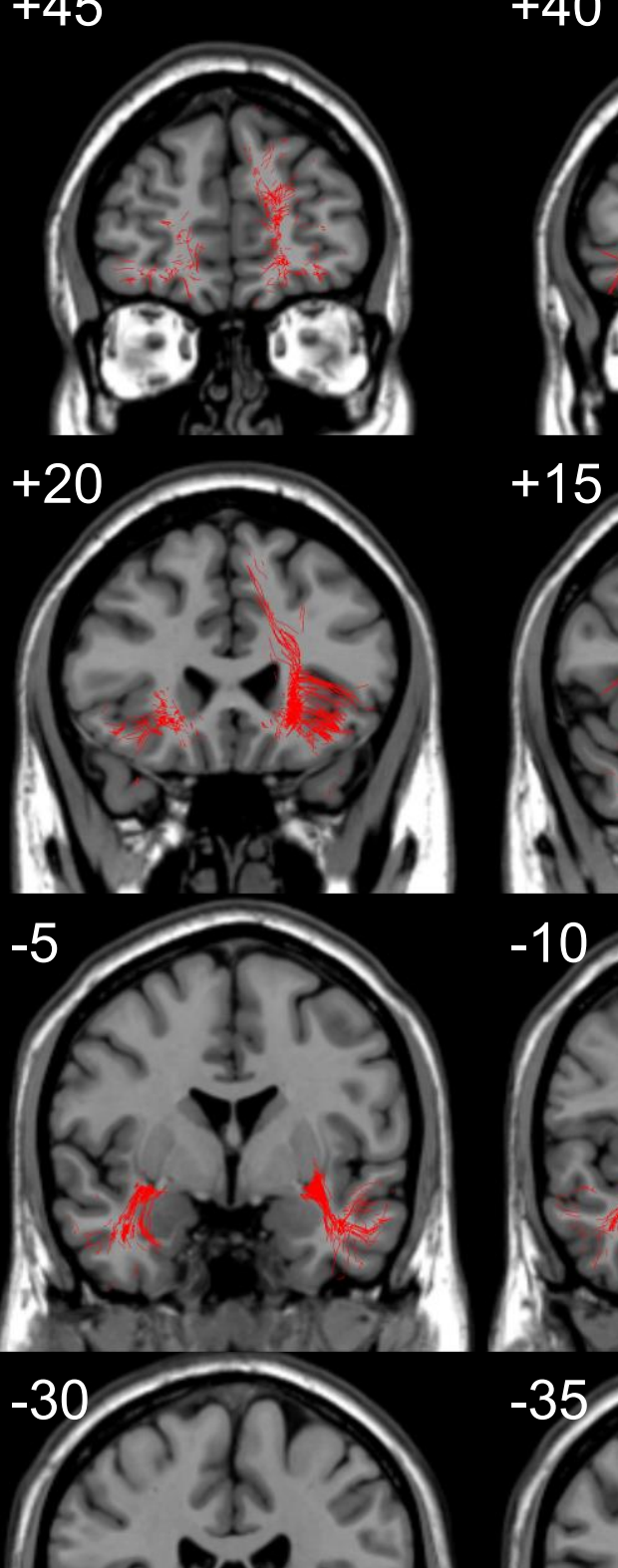

$-5$
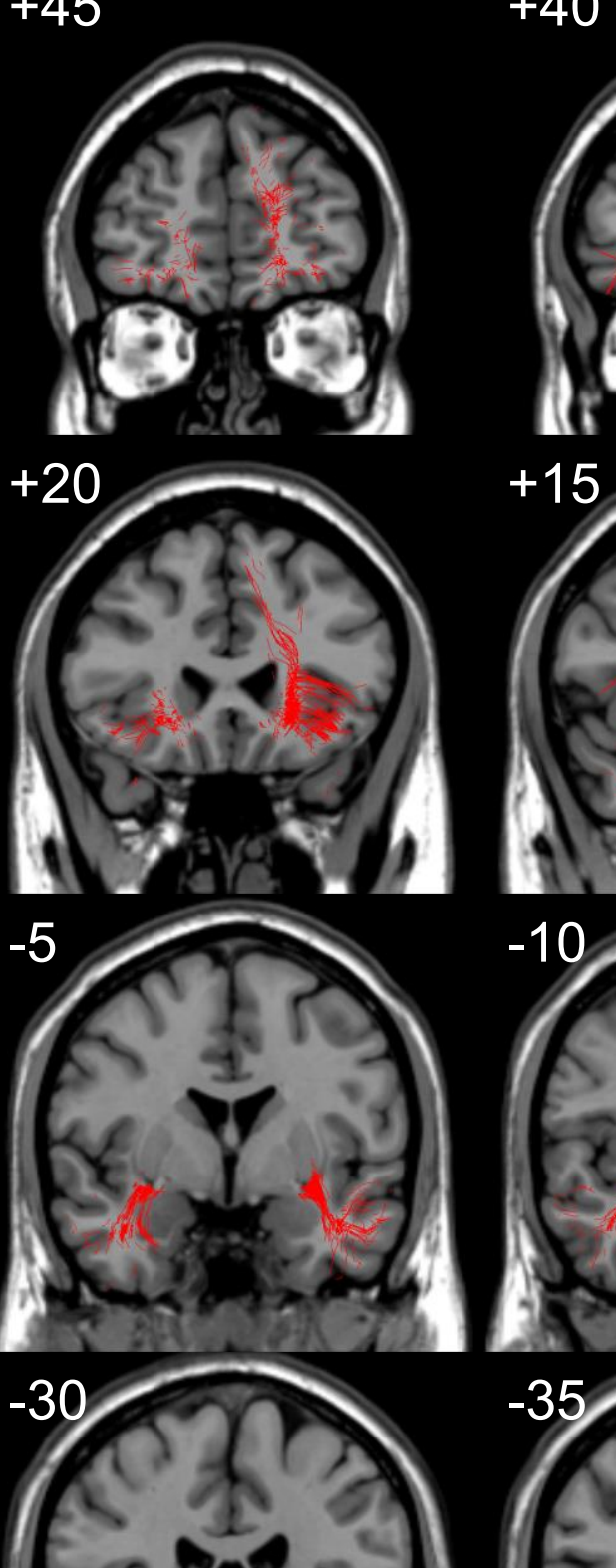

$-30$

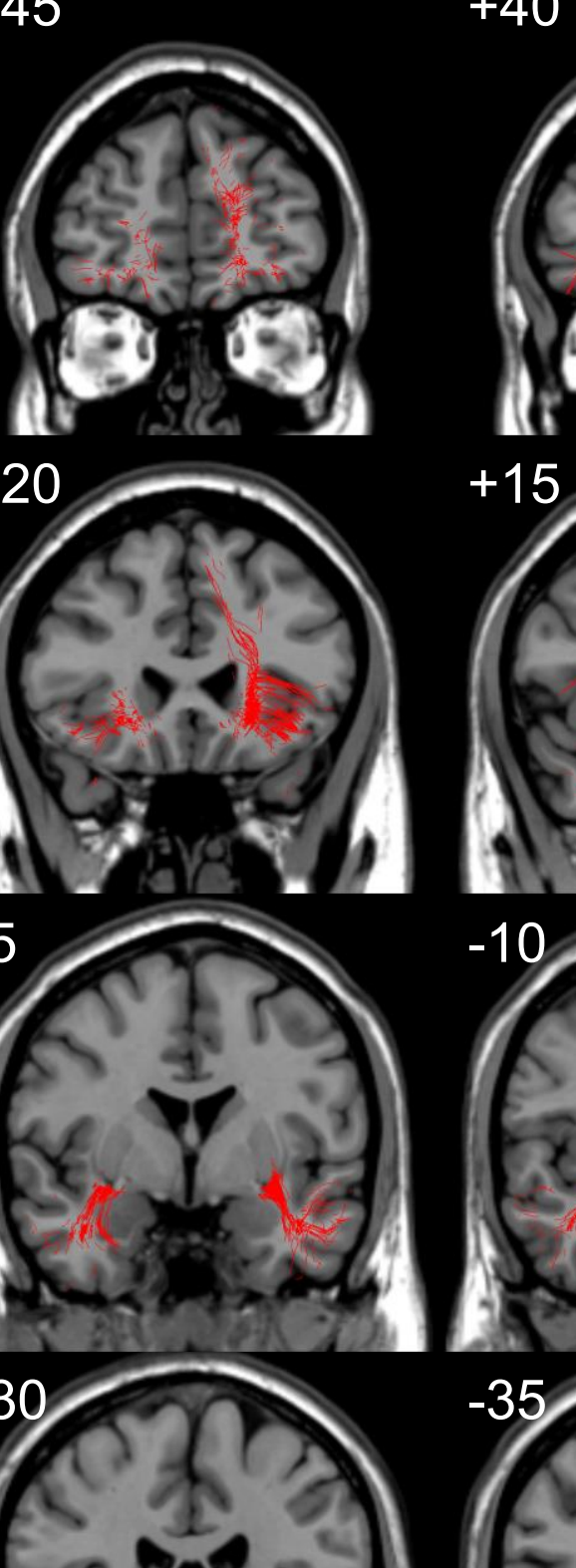

18

$\operatorname{lom}^{2} \mathrm{cos}$

3
$+40$

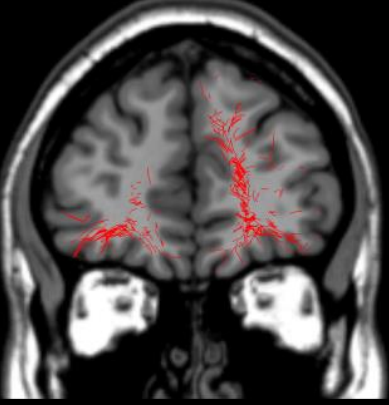

$+15$
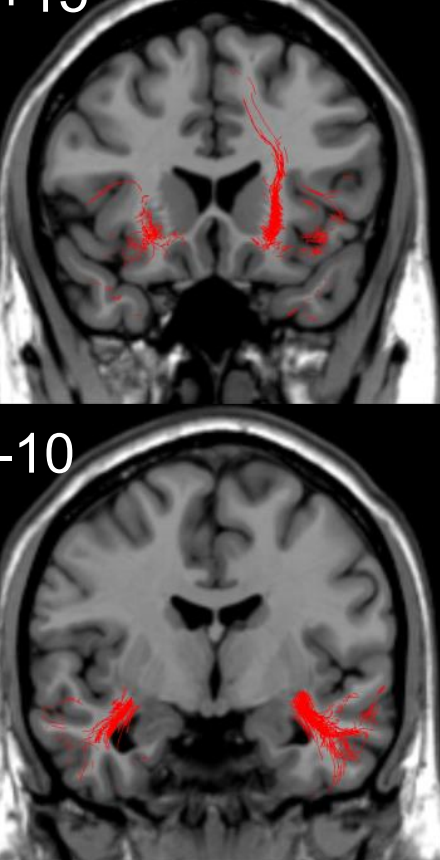

$\left(\begin{array}{ll}35 \\ x-2\end{array}\right.$
$+35$

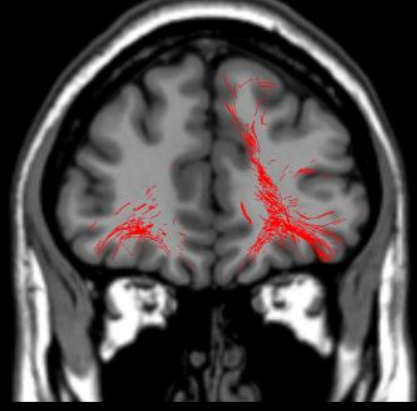

$+10$
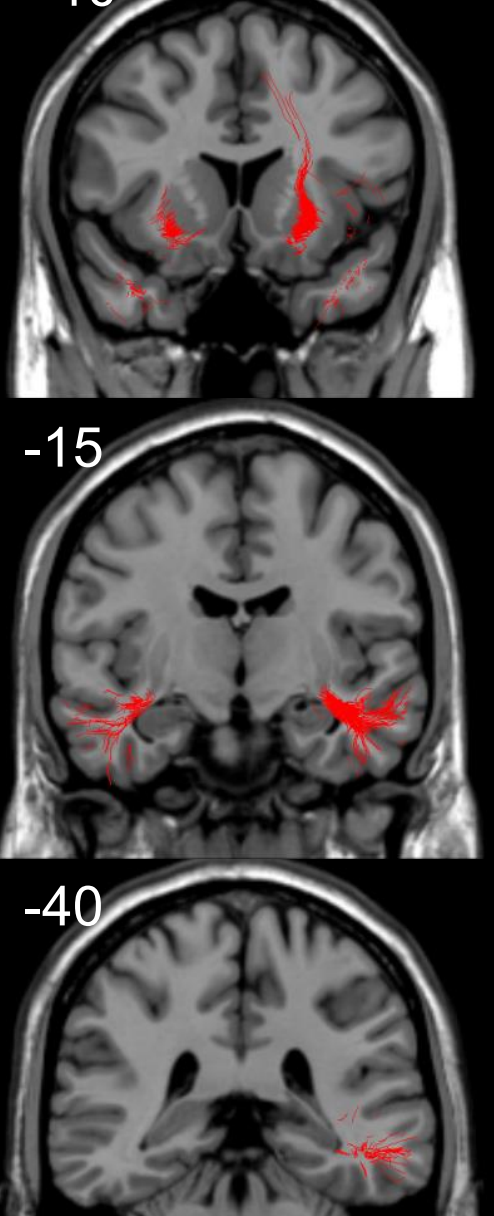

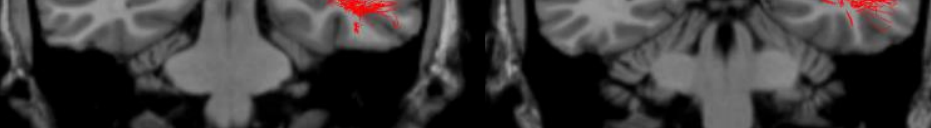

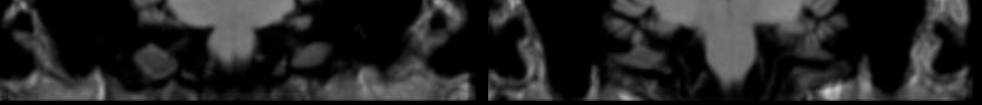


$+55$

R

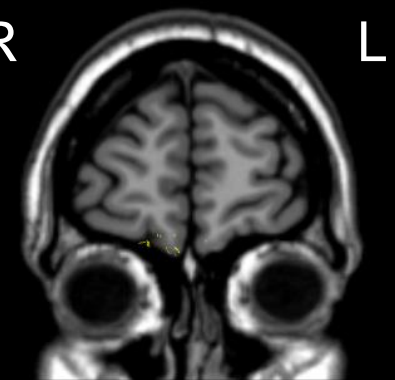

$+30$

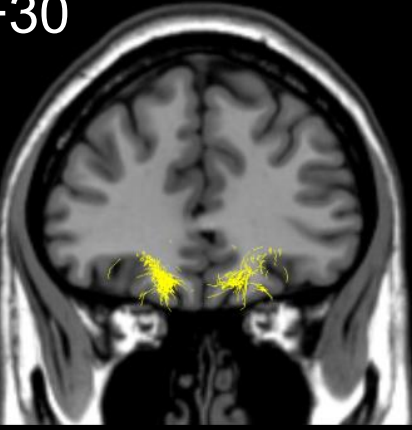

$+5$

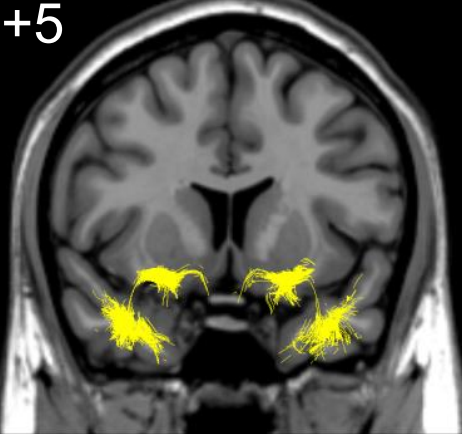

$-2$

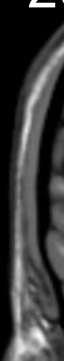

$+50$

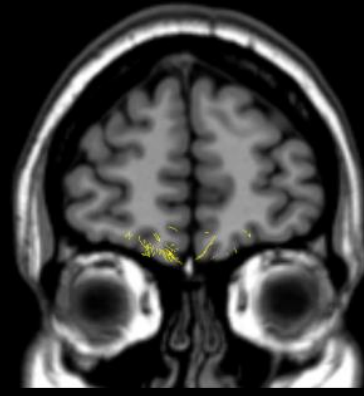

$+25$

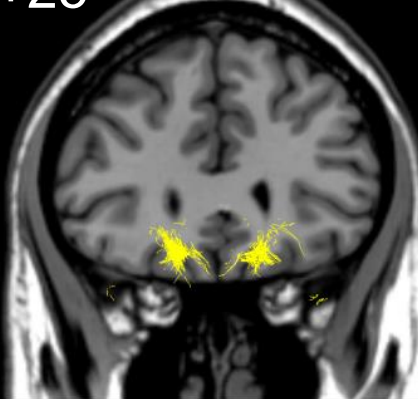

0
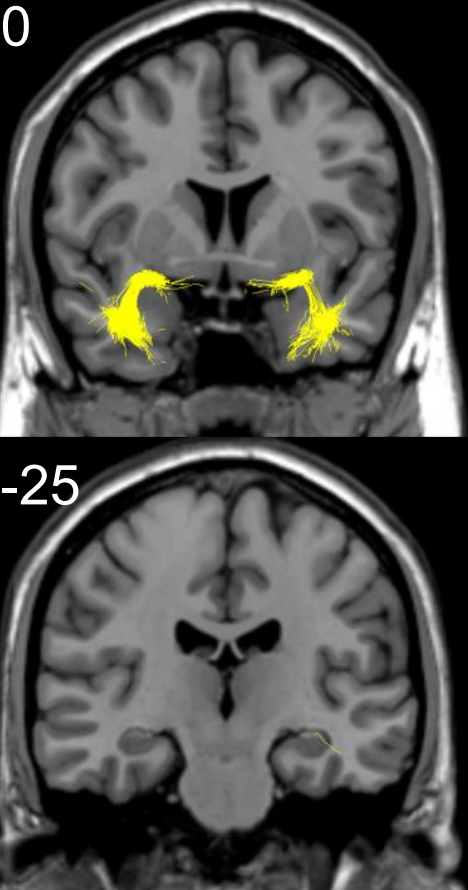

$+45$

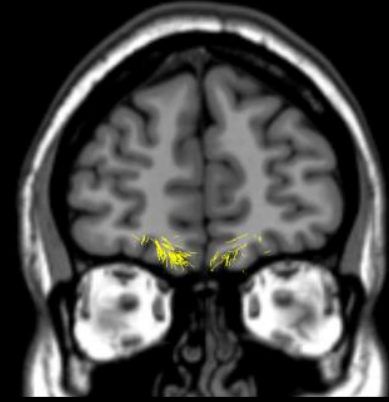

$+20$

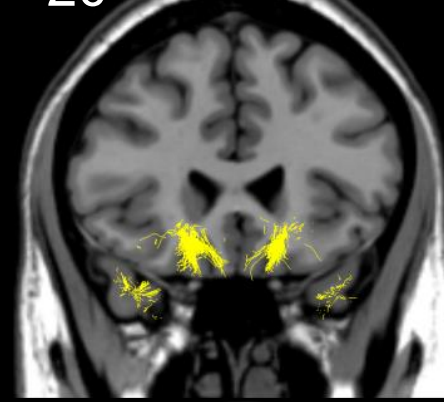

$-5$
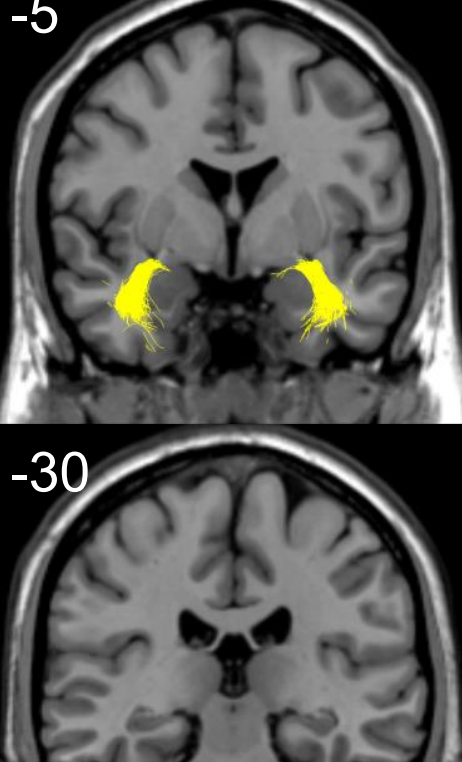

$\left(\begin{array}{ll}20 \\ x_{3}\end{array}\right.$
$+40$

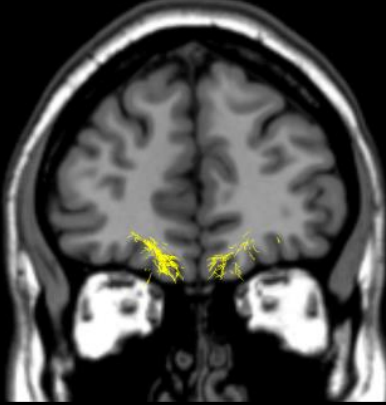

$+15$
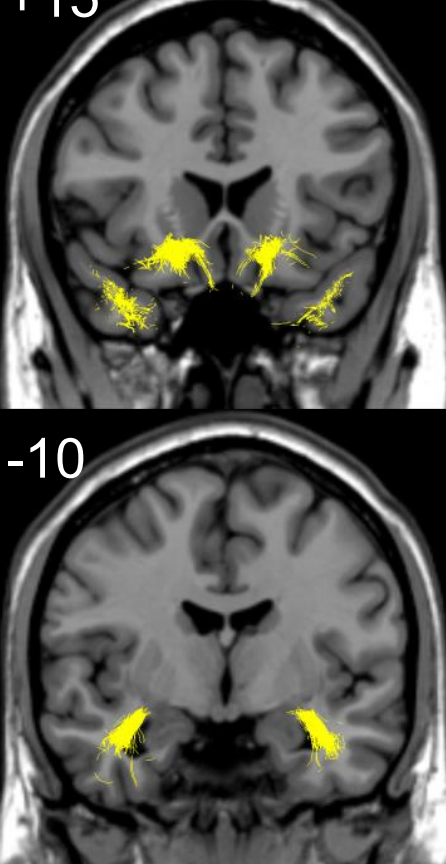

(c) of
$+35$

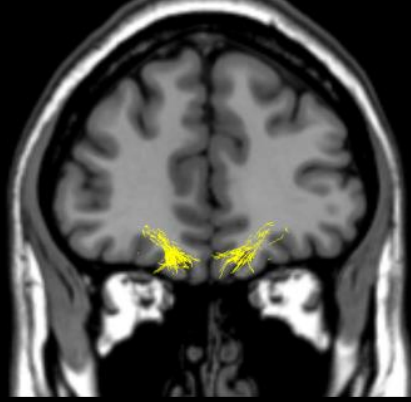

$+10$
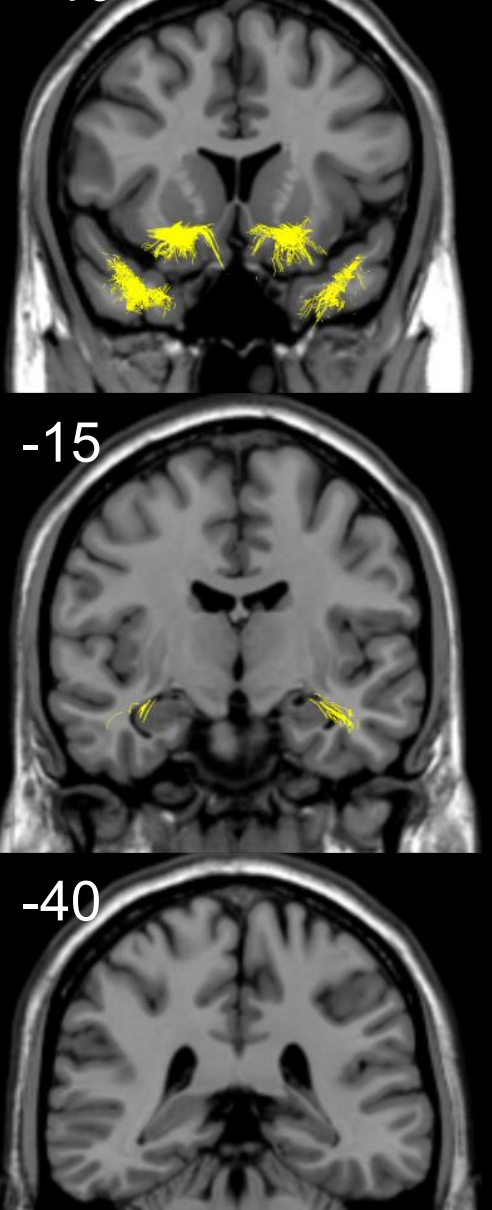
$+55$

R

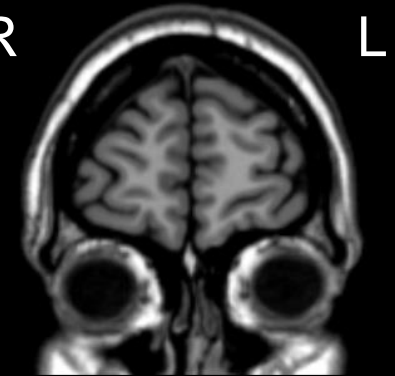

$+30$

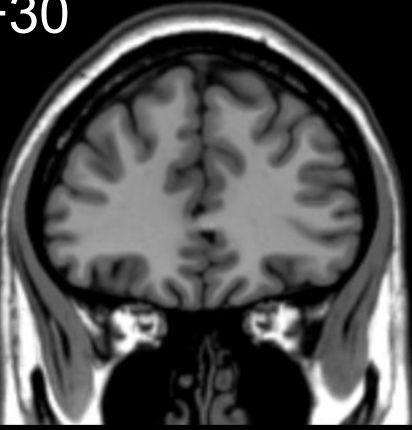

$+5$

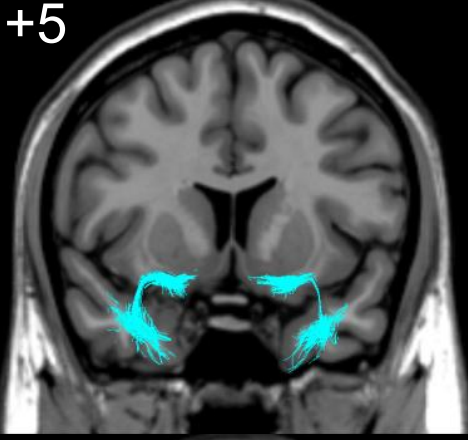

$-2$

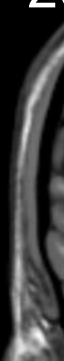

$+50$

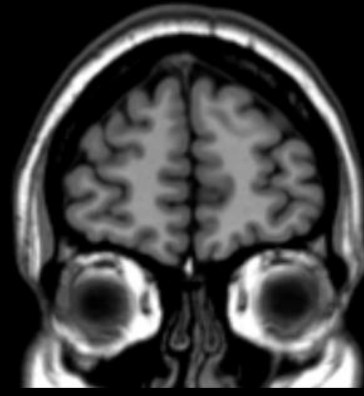

$+25$

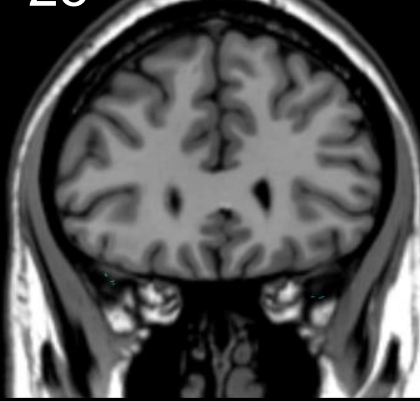

0
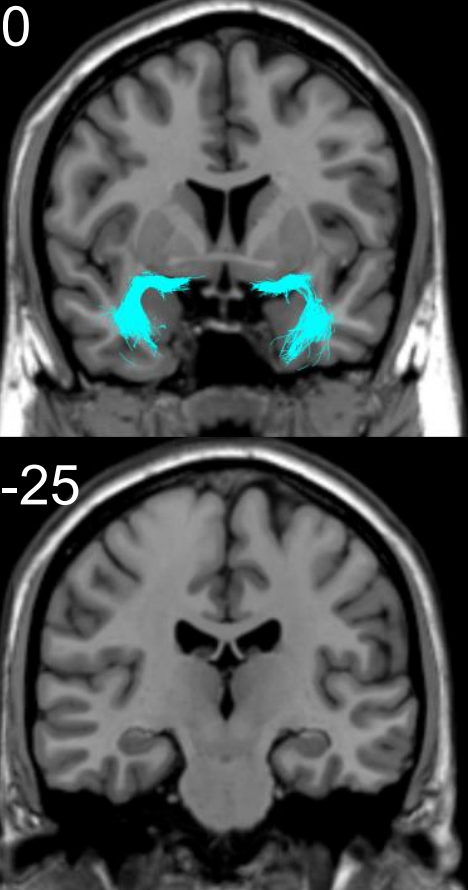

$+45$

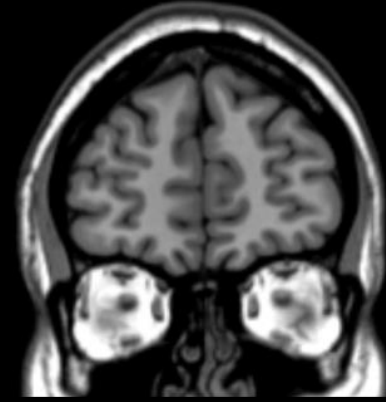

$+20$

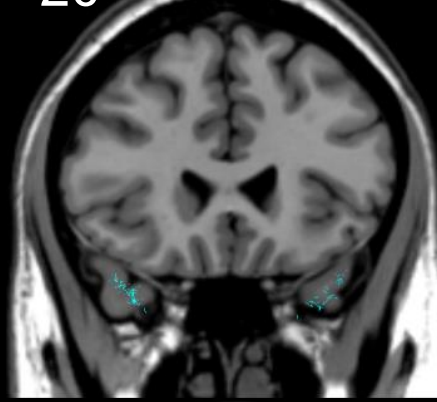

$-5$
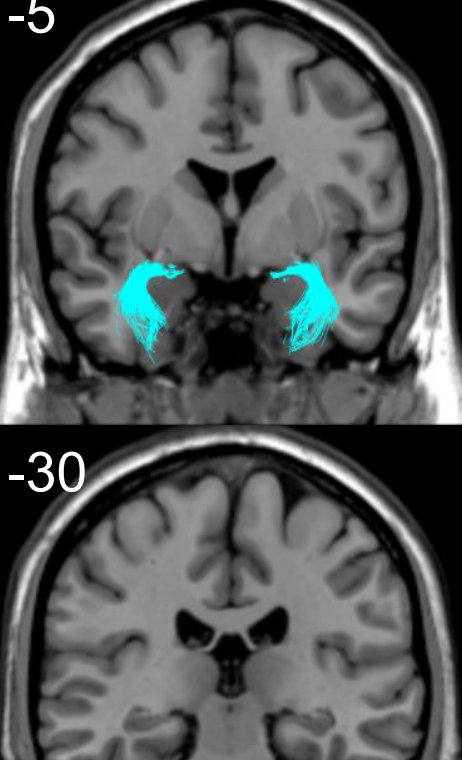

$+40$

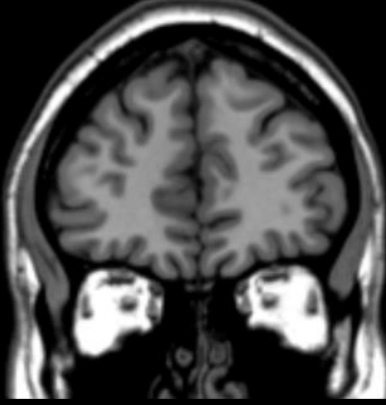

$+15$
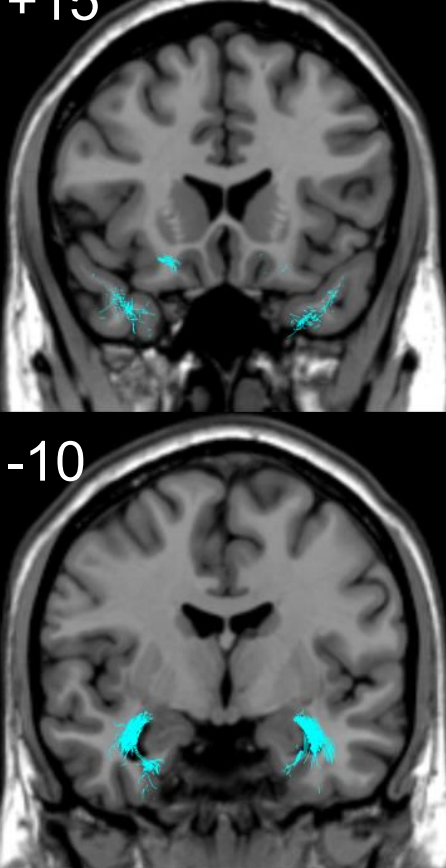

(c) of
$+35$

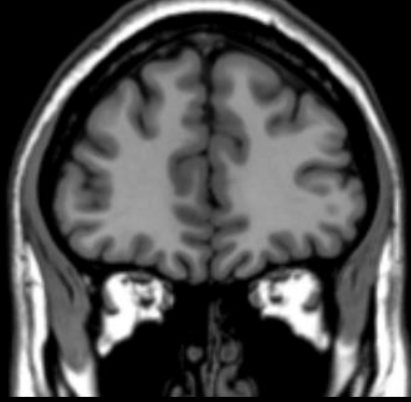

$+10$
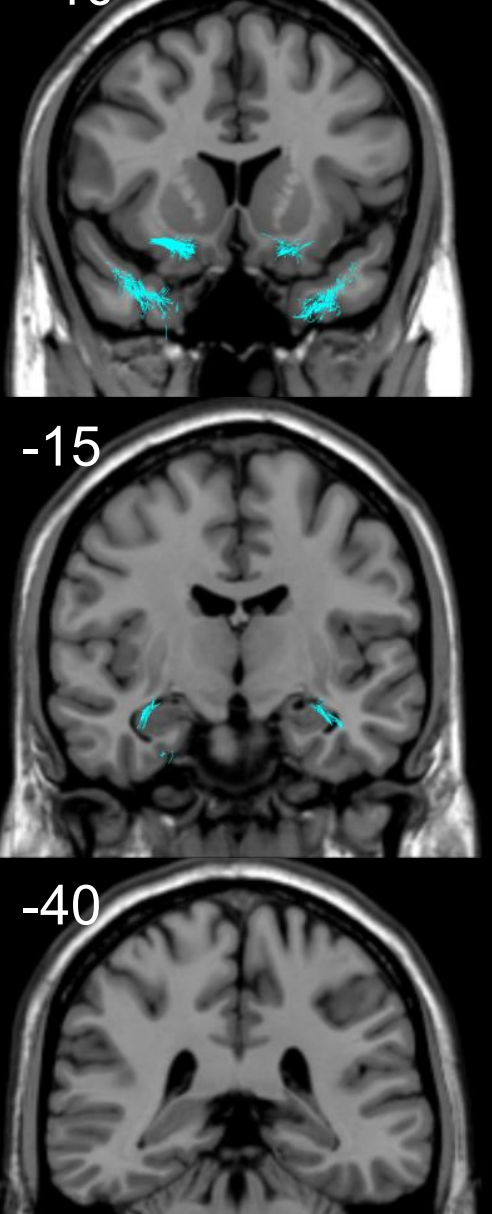
18.

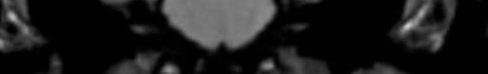
$2<33$

S. $\cos \theta$ a 
$+55$

R

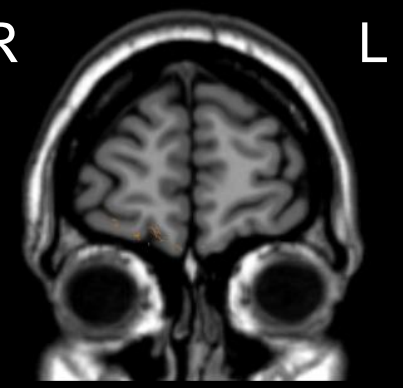

$+30$

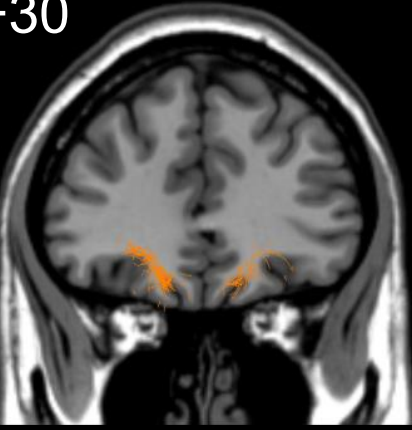

$+5$

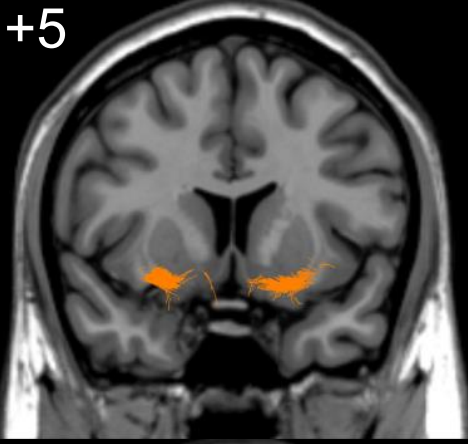

$-2$

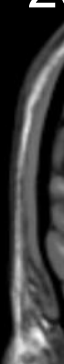

$+50$

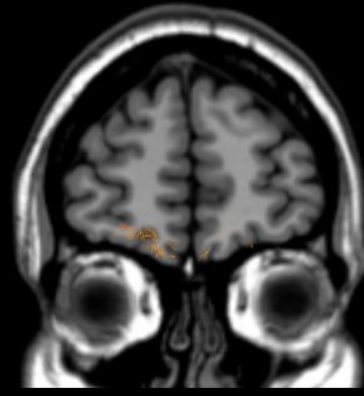

$+25$

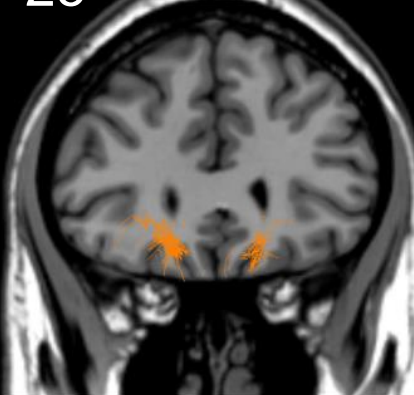

0

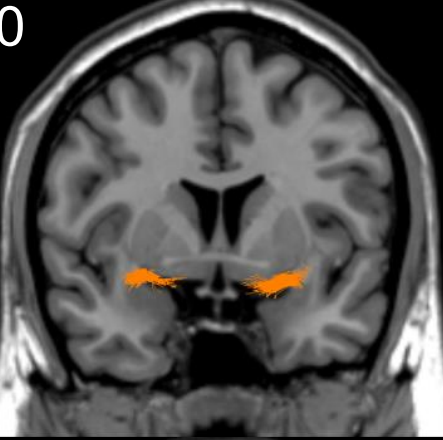

$-25$

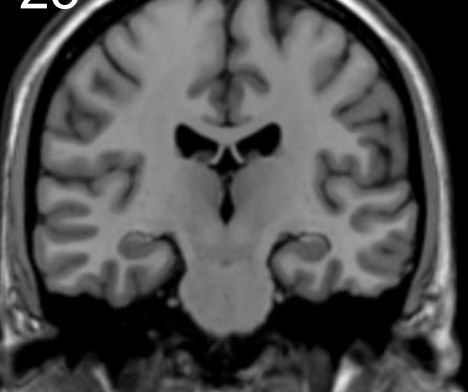

$+45$

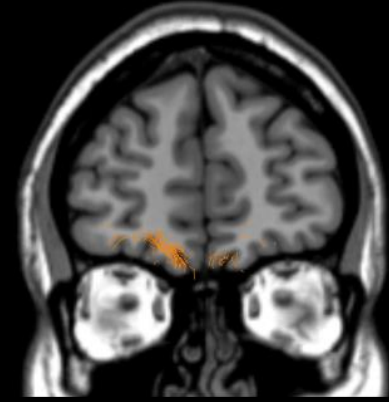

$+20$

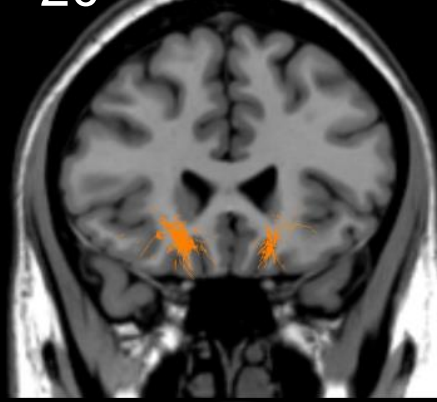

$-5$
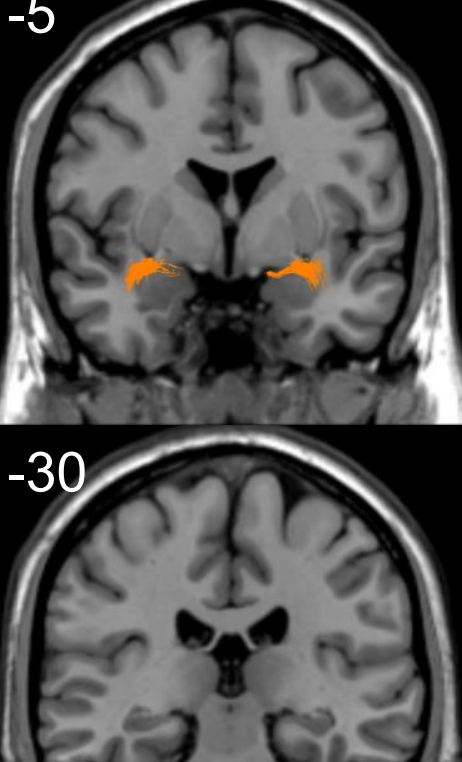

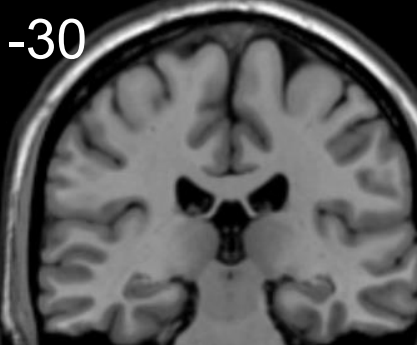

2
$+40$

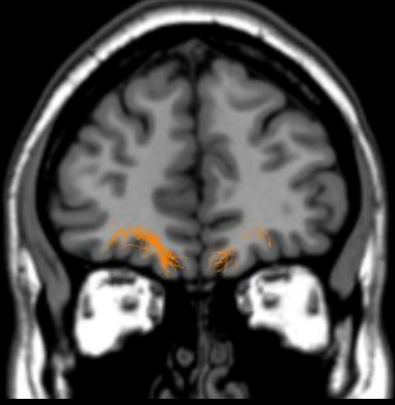

$+15$
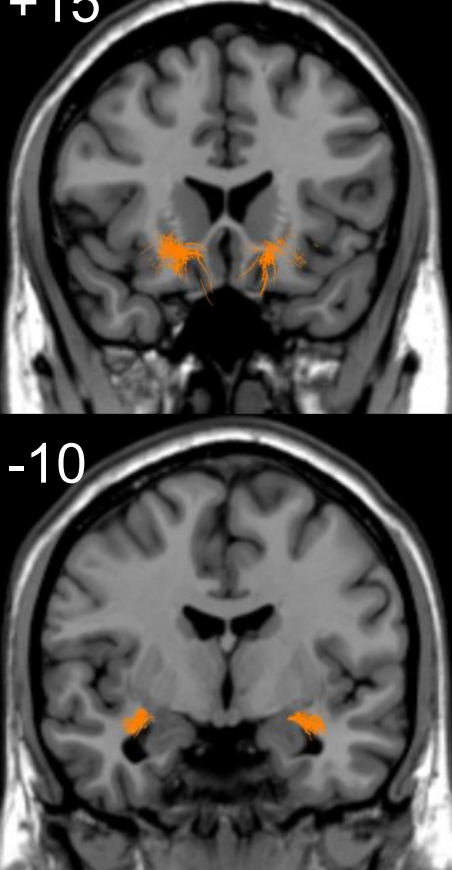

(c) of

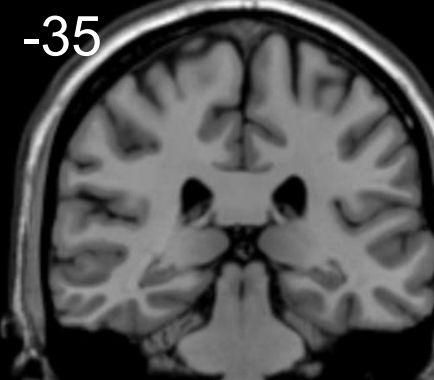

s.
$+35$

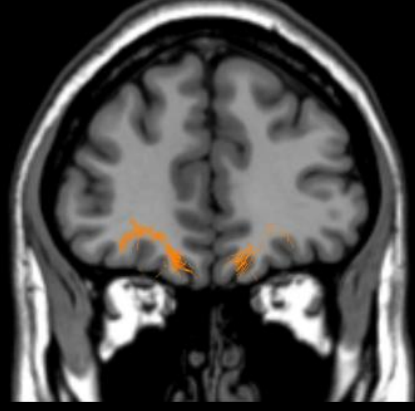

$+10$
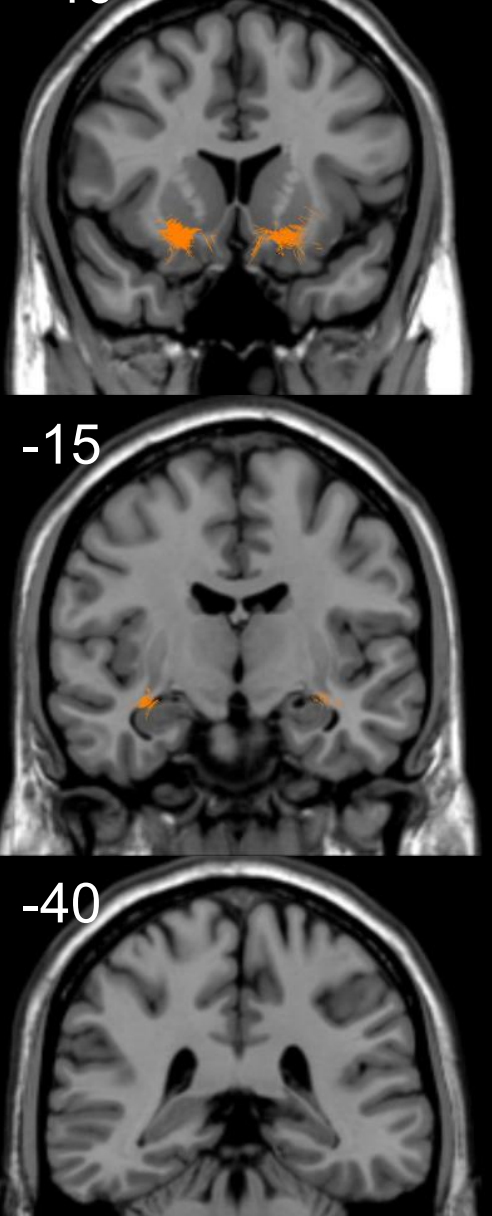